\title{
A Signaling Mechanism Coupling Netrin-1/Deleted in Colorectal Cancer Chemoattraction to SNARE-Mediated Exocytosis in Axonal Growth Cones
}

\author{
Tiziana Cotrufo, ${ }^{1 *}$ Francesc Pérez-Brangulí, ${ }^{1 *}$ Ashraf Muhaisen, ${ }^{1}$ Oriol Ros,,${ }^{1}$ Rosa Andrés, ${ }^{1}$ Thomas Baeriswyl, ${ }^{4}$ \\ Giulia Fuschini, ${ }^{1}$ Teresa Tarrago, ${ }^{2}$ Marta Pascual, ${ }^{1}$ Jesús Ureña, ${ }^{1}$ Joan Blasi, ${ }^{3}$ Ernest Giralt, ${ }^{2}$ Esther T. Stoeckli, ${ }^{4}$ \\ and Eduardo Soriano ${ }^{1}$ \\ ${ }^{1}$ Developmental Neurobiology and Regeneration Unit, Institut de Recerca Biomèdica (IRB) Barcelona, Parc Cientific de Barcelona, Centro de Investigación \\ Biomédica en Red sobre Enfermedades Neurodegenerativas (Instituto de Salud Carlos III), Department of Cell Biology, University of Barcelona, E-08028 Barcelona, \\ Spain, ${ }^{2}$ Design, Synthesis, and Structure of Peptides and Proteins, IRB Barcelona, Parc Cientific de Barcelona, Department of Organic Chemistry, University of \\ Barcelona, E-28028 Barcelona, Spain, ${ }^{3}$ Department of Experimental Pathology, University of Barcelona, E-08028 Barcelona, Spain, and ${ }^{4}$ Institute of Molecular Life \\ Sciences, University of Zurich, CH-8057 Zurich, Switzerland
}

Directed cell migration and axonal guidance are essential steps in neural development. Both processes are controlled by specific guidance cues that activate the signaling cascades that ultimately control cytoskeletal dynamics. Another essential step in migration and axonal guidance is the regulation of plasmalemma turnover and exocytosis in leading edges and growth cones. However, the cross talk mechanisms linking guidance receptors and membrane exocytosis are not understood. Netrin-1 is a chemoattractive cue required for the formation of commissural pathways. Here, we show that the Netrin-1 receptor deleted in colorectal cancer (DCC) forms a protein complex with the t-SNARE (target SNARE) protein Syntaxin-1 (Sytx1). This interaction is Netrin-1 dependent both in vitro and in vivo, and requires specific Sytx1 and DCC domains. Blockade of Sytxl function by using botulinum toxins abolished Netrin-1-dependent chemoattraction of axons in mouse neuronal cultures. Similar loss-offunction experiments in the chicken spinal cord in vivo using dominant-negative Sytx1 constructs or RNAi led to defects in commissural axon pathfinding reminiscent to those described in Netrin-1 and DCC loss-of-function models. We also show that Netrin-1 elicits exocytosis at growth cones in a Sytx1-dependent manner. Moreover, we demonstrate that the Sytx1/DCC complex associates with the v-SNARE (vesicle SNARE) tetanus neurotoxin-insensitive vesicle-associated membrane protein (TI-VAMP) and that knockdown of TI-VAMP in the commissural pathway in the spinal cord results in aberrant axonal guidance phenotypes. Our data provide evidence of a new signaling mechanism that couples chemotropic Netrin-1/DCC axonal guidance and Sytx1/TI-VAMP SNARE proteins regulating membrane turnover and exocytosis.

\section{Introduction}

Netrin-1 is a chemoattractive and chemorepellent cue required for the formation of commissural pathways throughout the brain and spinal cord (Kennedy et al., 1994; Serafini et al., 1994, 1996; Fazeli et al., 1997; Barallobre et al., 2000). Deleted in colorectal

\footnotetext{
Received June 10, 2011; revised July 27, 2011; accepted Aug. 12, 2011.

Author contributions: T.C., F.P.-B., E.T.S., and E.S. designed research;T.C., F.P.-B., A.M., O.R., R.A., T.B., G.F., T.T., M.P., J.U., J.B., E.G., and E.T.S. performed research; J.B. contributed unpublished reagents/analytic tools; T.C., F.P.-B., A.M., O.R., R.A., T.B., G.F., T.T., M.P., J.U., J.B., E.G., and E.T.S. analyzed data; T.C., F.P.-B., E.T.S., and E.S. wrote the paper.

This work was supported by grants from the Ministerio de Ciencia e Innovación (MICINN), La Caixa Foundation, Marató de TV3 Foundation, Fondo de Investigación Sanitaria (Instituto de Salud Carlos III), and Caixa Catalunya Foundation (awarded to E.S., J.U., and M.P.), from the MICINN (J.B. and E.G.), and from the National Swiss Foundation (E.T.S.). We thank M. Popoff (Paris, France), T. Galli (Paris, France), M. Fukuda (RIKEN), E. Stein (Yale University, New Haven, (T), and J. Rizo, T. Südhof, and I. Dulubova (University of Texas Southwestern Medical Center, Dallas, TX) for reagents. We thank Hagar Lock and Maica López for technical support, Fernando Aguado for valuable discussion, and Robin Rycroft and Tanya Yates for editorial assistance.

The authors declare no competing financial interests.

${ }^{*}$ T.C. and F.P.-B. contributed equally to this work.

Correspondence should be addressed to Dr. Eduardo Soriano, Institut de Recerca Biomèdica Barcelona, Baldiri Reixac 10, E-08028 Barcelona, Spain. E-mail: eduardo.soriano@irbbarcelona.org.

DOI:10.1523/JNEUROSCI.3018-11.2011

Copyright $\odot 2011$ the authors $\quad 0270-6474 / 11 / 3114463-18 \$ 15.00 / 0$
}

cancer (DCC), DSCAM (Down syndrome cell adhesion molecule), and the UNC5 family of proteins are Netrin-1 receptors that mediate chemoattraction and chemorepulsion, respectively (Keino-Masu et al., 1996; Fazeli et al., 1997; Hong et al., 1999; Ly et al., 2008). Netrin-1 signaling activates several kinases (Forcet et al., 2002; Ren et al., 2004) and the responses to Netrin-1 are regulated by cyclic nucleotides and the activation of L-type $\mathrm{Ca}^{2+}$ channels (Song and Poo, 2001; Nishiyama et al., 2003). Moreover, Netrin-1 signaling is linked to cytoskeletal rearrangement (Del Río et al., 2004; Dent et al., 2004), and it was recently shown that asymmetrical $\beta$-actin translation mediates responses to Netrin-1 (Leung et al., 2006; Tcherkezian et al., 2010).

Synaptic vesicle exocytosis is controlled by a complex protein machinery. Neurotransmitter secretion depends on the assembly of the soluble $N$-ethylmaleimide-sensitive factor attachment protein receptor (SNARE) complex formed by the proteins Syntaxin 1 (Sytx1), synaptosomal-associated protein of $25 \mathrm{kDa}$ (SNAP25) [plasma membrane target SNAREs (t-SNAREs)], and vesicleassociated membrane protein 2 (VAMP2)/Synaptobrevin [vesicle SNARE (v-SNARE)] (Rizo and Südhof, 2002; Jahn and Scheller, 2006; Südhof and Rothman, 2009), as well as on the participation of 
other proteins, such as the small GTPases Rabs, Complexins, Synaptotagmins, MUNC-18, and Rab3a-interacting molecule (RIM) (Verhage et al., 2000; Castillo et al., 2002; de Wit et al., 2009; Maximov et al., 2009). SNARE proteins are essential for calciumregulated exocytosis in neurons, and for a variety of cellular processes involving membrane fusion events (Rizo and Südhof, 2002; Jahn and Scheller, 2006; Südhof and Rothman, 2009). In addition to expressing the machinery required for axonal guidance, the growth cone is believed to be a major site of exocytosis, thereby allowing the extension of axons. In fact, many proteins involved in exocytosis have been localized in developing growth cones (Igarashi et al., 1997; Martinez-Arca et al., 2001; Sabo and McAllister, 2003; Alberts et al., 2006; Tojima et al., 2007; Pfenninger, 2009). It is conceivable that axonal guidance may rely on the precise control of exocytosis at select regions of the growth cone. For instance, chemoattractant guidance cues may lead to increased exocytosis, thereby resulting in membrane extension in a given direction of growth. Recent studies have shown that PKA (protein kinase A) activation recruits DCC to the plasma membrane and enhances axon chemoattraction to Netrin-1 (Bouchard et al., 2004; Moore et al., 2008). Indeed, recent studies showed that local increases of $\mathrm{Ca}^{2+}$ result in asymmetric exocytosis in the growth cone (Tojima et al., 2007) and that clathrin-mediated endocytosis is essential for Semaphorin 3A-induced chemorepulsion (Tojima et al., 2010). Here, we studied the molecular cross talk between Netrin-1 chemoattraction and SNARE proteins. We show that the DCC receptor associates specifically with the SNARE proteins Sytx 1 and tetanus neurotoxininsensitive vesicle-associated membrane protein (TI-VAMP) and that this association is Netrin-1 dependent. Furthermore, interference with Sytxl function blocks Netrin-1-mediated chemoattraction of axons.

\section{Materials and Methods}

Materials. Botulinum neurotoxin type A (BoNT/A) was generated as described previously (Blasi et al., 1993a). BoNT/C1 was generously provided by M. R. Popoff (Institut Pasteur, Paris, France). Tetanus neurotoxin (TeNT) was purchased from Calbiochem. Rat pRc/CMVSytx1A was cloned as described previously (Paradisi and Mehlen, 2010). Fulllength Sytx1A or truncated DNAs (Sytx1ACYT, Sytx1AHabc, Sytx1AH3TM, Sytx1AH3, and Sytx1ATM) were cloned into EcoRI sites in pEGFPC1 fusion mammalian protein expression vector (Clontech). The truncated fragments were obtained by PCR amplification of rat Sytx 1 A using the following primers: $5^{\prime}$-GAATTCTATGAAGGACCGAA C-3' and 5'-GGGCCCCTACGTGGTCCG-3' for Sytx1AHabc, 5' -GAAT TCTATGATCATCATGGA- $3^{\prime}$ and $5^{\prime}$-GGGCCCGATCTATCCAAAGA T-3' for Sytx1AH3TM, 5'-ATGAAGGACCGAACCCGA-3' and 5'-CT ACTTCTTCCTGCGTGC-3' for Sytx1ACYT, $5^{\prime}$-ATGATCATCATGGA CTCCAGC- $3^{\prime}$ and $5^{\prime}$-CTACTTCTTCCTGCGTGCCTT- $3^{\prime}$ for Sytx1AH3, and $5^{\prime}$-ATGATCATGATCATCATTTGC- ${ }^{\prime}$ ' and $5^{\prime}$-CTAGATCTATTCAA AGATGCC-3' for Sytx1ATM. The Sytx1AH3TM fragment was also cloned in the EcoRI site in PIRES2EGFP mammalian protein expression vector (Clontech).

pCDNA3-hDCC was generously provided by E. Stein (Yale University, New Haven, CT). The truncated fragments were obtained by PCR amplification of human DCC using the following primers: $5^{\prime}$-TGCACCCG ACGC-3' and 5' -AAAGGCTGAGCCTG-3' for DCCT1-P3, 5' -AACAGC AACCTG-3' and 5'-AAAGGCTGAGCCTG-3' for DCCTM-P3, 5' -AA CAGCAACCTG- $3^{\prime}$ and $5^{\prime}$-TGTTGGCACAGA-3' for DCCTM-T3, 5' AACAGCAACCTG- $3^{\prime}$ and $5^{\prime}$-TGGTGCCTCCTCGC- ${ }^{\prime}$ ' for DCCTMT2, $5^{\prime}$-ATGTGCACCCGACGCTCTTTCA- $3^{\prime}$ and $5^{\prime}$-TTCAATATTTTT CATCTC-3' for DCCT1-P1, 5'-ATGTGCACCCGACGCTCTTTCA-3' and $5^{\prime}$-TCGGAGGTCCTTCTG-3' for DCCT1, and 5' -ATGCCCCCTG ATCTTTGGATC-3' and 5'-AAAGGCTGAGCCTG-3' for DCCP1-P3. Truncated DCC DNAs (DCCT1-P3, DCCTM-P3, DCCTM-T3, DCCTM-T2, DCCT1-P1, DCCT1, DCCP1-P3) were cloned into HindIII sites of the pEGFPC3 expression vector (Clontech). TI-
VAMPpCMV6 was obtained from Origene. pEF-BOS-SNAP25-FLAG and pEF-BOS-VAMP2-FLAG plasmids were kindly provided by Dr. M. Fukuda (RIKEN, Wako, Saitama, Japan).

Animals. OF1 embryos and postnatal and adult mice (Iffa Credo) were used. The mating day was considered as embryonic day 0 (E0), and the day of birth, as postnatal day 0 (P0). Adult heterozygous mice carrying a mutation in the netrin-1 gene, provided by $\mathrm{M}$. Tessier-Lavigne (Rockefeller University, New York, NY), were crossed, and the offspring were genotyped as described previously (Serafini et al., 1996). Adult mice were killed in accordance with National and European regulations.

Production of recombinant Netrin-1. Netrin-1-conditioned media was prepared by culturing HEK293 cells that had been stably transfected with pCEP4-rNetrin-1c-myc or with pCEP451 at $60-80 \%$ confluence. Cell culture medium was collected after $2 \mathrm{~d}$, filtered, and concentrated through a $0.20 \mu \mathrm{m}$ NC filter (Millipore). The presence of rNetrin-1-myc in the media was checked by Western blot (WB) with an $\alpha$-Myc antibody (1:1000; Roche). Netrin-1- and control-conditioned media were used at a dilution of 1:15/20.

Cell transfection. HEK293 or COS- 1 cells were plated at $40-60 \%$ confluence the day before transfection. Transfection was performed using Lipofectamine Plus reagent (Invitrogen). Cells were transfected with different combinations of DNAs. For immunoprecipitation assays, 3-4 $\mu \mathrm{g}$ of DNA per plate was used in $90 \mathrm{~mm}$ culture plates. For the immunofluorescence analyses, $0.4 \mu \mathrm{g}$ of DNA per well was used in 24-well culture dishes.

Dissociated and explant cultures. The hippocampus of E15 mouse embryos was dissected out to obtain primary neuronal cultures. After trypsin (Invitrogen) and DNase (Roche Diagnostics) treatments, tissue was dissociated by gentle sweeping. Cells were cultured for 2-4 d onto polyD-lysine-coated dishes in Neurobasal medium containing L-glutamine and B27 supplement (Invitrogen). For biochemical analyses, cells were plated at a concentration of 5-8 million cells per $90 \mathrm{~mm}$ plate. For immunofluorescence, 50,000 cells were plated onto coverslips. Primary cultures were incubated with Netrin-1-containing or control media, for 15-30 min, or with BDNF (50 ng/ml; Promega). Cultures were also treated with $25 \mathrm{~nm}$ BoNT/A, $15 \mathrm{~nm} \mathrm{BoNT/C1}$, or $2 \mathrm{~nm}$ TeNT, 2-3 h before the addition or Netrin-1 or BDNF.

To obtain hippocampal explants, brains (E15) were sectioned using a tissue chopper. Small tissue explants (300-400 $\mu \mathrm{m}$ thick) were obtained from the CA1/CA3 regions (Barallobre et al., 2000). Tissue explants were cocultured at a distance $(500-600 \mu \mathrm{m})$ with aggregates of HEK293 cells stably transfected with a pCEP4-rNetrin-1c-myc construct or with control cells. Explants and cell aggregates were embedded in collagen gels and cultured for $2 \mathrm{~d}$. Hippocampal explants were cultured in Neurobasal medium plus $0.54 \%$ glucose, $0.032 \% \mathrm{NaH}_{2} \mathrm{CO}_{3}$, L-glutamine, $10 \%$ horse serum, and supplemented with B27. Cultures were treated with $25 \mathrm{~nm}$ BoNT/A, 15 nм BoNT/C1, or 2 nм TeNT.

Immunoprecipitation assays and immunoblots. Embryonic, postnatal, and adult brains, as well as primary cultures and transiently transfected HEK293 or COS-1 cells were lysed in hypotonic buffer [ $150 \mathrm{~mm} \mathrm{NaCl}, 50$ mм Tris, pH 7.2, 2 mм EDTA, 1\% Triton X-100, protease inhibitors (Complete; Mini Protease Inhibitor Cocktail Tablets; Roche)]. For the immunoprecipitation assays, $100-200 \mu \mathrm{g}$ of total protein per sample were used.

Brain and primary culture lysates were incubated overnight at $4^{\circ} \mathrm{C}$ with mouse $\alpha$-DCC (1:100; Oncogene), $\alpha$-Sytx1 (HPC-1 clone; 1:5001000; Sigma-Aldrich), $\alpha$-SNAP25 (SMI-81 clone; 1:500; SternbergerMeyer), $\alpha$-VAMP2 (1:500; Synaptic Systems), $\alpha$-TI-VAMP (clone 158.2; 1:500; Abcam), or $\alpha$-Sytx 4 (1:500; Synaptic Systems) in immunoprecipitation buffer (10 mm Tris, pH 7, $140 \mathrm{~mm} \mathrm{NaCl}, 1.5 \mathrm{~mm}$ EDTA). Transfected HEK293 or COS-1 cell homogenates were incubated with $\alpha$-DCC, $\alpha$-Sytx1, $\alpha$-TI-VAMP, $\alpha$-myc (1:500; Roche), $\alpha$-HA (1:500; Roche), or $\alpha$-GFP (1:1000; Invitrogen) antibodies. All the assays were performed using protein G-Sepharose beads (Sigma-Aldrich) for $2 \mathrm{~h}$ at $4^{\circ} \mathrm{C}$.

After three washes with buffer ( $10 \mathrm{~mm}$ Tris, $\mathrm{pH} 7,500 \mathrm{~mm} \mathrm{NaCl}, 1 \mathrm{~mm}$ EDTA, 1 mm EGTA, 1\% Triton X-100, 0.5\% NP-40), SDS-sample buffer was added to the beads, and the proteins were analyzed by SDS-PAGE and WB. Proteins were transferred onto nitrocellulose membranes, which were blocked with $5 \%$ nonfat dry milk in Tris-HCl-buffered saline (TBS) containing $0.1 \%$ Tween 20 , and incubated overnight at $4^{\circ} \mathrm{C}$ with 
mouse $\alpha$-Sytx1 (1:1000), mouse $\alpha$-DCC (1:100), $\alpha$-SNAP25 (1:1000), $\alpha$-VAMP2 (1:4000), rabbit $\alpha$-Sytx 4 (1:1000), $\alpha$-TI-VAMP (1:500), $\alpha$-HA (1:1000), or rabbit $\alpha$-GFP (1:2000) antibodies. After incubation with secondary antibodies, blots were developed following the enhanced chemiluminescence (ECL) method (GE Healthcare). Sytx1A and 1B isoforms were analyzed by urea/SDS-PAGE, and the WBs were probed with the $\alpha$-Sytx 1 antibody. WBs were quantified (in arbitrary units; GelPro Analyzer 3.1; Media Cybernetics). To normalize the data, we represented the ratios between the amount of coimmunoprecipitated protein (e.g., Sytx1) and the immunoprecipitating protein (DCC) (expressed as percentage association, respect to controls).

Sytx $1 \mathrm{~A}$ expression and purification and affinity pull-down assays. Tetanus toxin heavy chain (HC-TeTx) and prolyl oligopeptidase (POP) were obtained by expression in Escherichia coli and affinity purification using a His tail fusion as reported previously (Gil et al., 2001; Tarrago et al., 2006). E. coli Rosetta BL21 (DE3) competent cells were transformed with pET28aHisSytx1A plasmid (gift from Dr. J. Rizo-Rey, Dallas, TX). The autoinduction protocol was used to produce the expression of the recombinant protein (Studier, 2005). Briefly, the inoculated cultures were grown at $37^{\circ} \mathrm{C}$ during $2 \mathrm{~h}$, and afterward the temperature was decreased to $16^{\circ} \mathrm{C}$; the autoinduction incubation was performed for $30 \mathrm{~h}$. Cells were harvested $\left(3500 \times g, 15 \mathrm{~min}, 4^{\circ} \mathrm{C}\right)$ and the pellet was suspended in suspension buffer $(50 \mathrm{ml})(50 \mathrm{~mm}$ Tris- $\mathrm{HCl}, \mathrm{pH} 8,300 \mathrm{~mm} \mathrm{NaCl}, 5 \mathrm{~mm}$ imidazole, $0.5 \%$ Triton X-100, protease inhibitors without EDTA). After sonication, the sample was centrifuged $\left(40,000 \times g, 30 \mathrm{~min}, 4^{\circ} \mathrm{C}\right)$, and the supernatant was used immediately for His-Sytx1A purification. An ÄKTA explorer FPLC system was used for purification (GE Healthcare). The supernatant was applied at a flow of $1 \mathrm{ml} / \mathrm{min}$ to a HiTrapQuelating column ( $5 \mathrm{ml}$ ) (GE Healthcare). The column was washed with suspension buffer until the absorbance at $280 \mathrm{~nm}$ returned to basal level. The column was then rinsed with 5 vol of washing buffer $(50 \mathrm{~mm}$ Tris- $\mathrm{HCl}$, pH 8, $300 \mathrm{~mm} \mathrm{NaCl}, 50 \mathrm{~mm}$ imidazole, $0.5 \%$ Triton X-100, protease inhibitors without EDTA). The elution was performed with 3 vol of elution buffer ( $50 \mathrm{~mm}$ Tris- $\mathrm{HCl}, \mathrm{pH} 8,300 \mathrm{~mm} \mathrm{NaCl}, 500 \mathrm{~mm}$ imidazole, $0.5 \%$ Triton X-100, protease inhibitors without EDTA). Fractions ( $4 \mathrm{ml})$ were collected during the entire elution.

The presence of His-Sytx1A was checked in all fractions by SDS-PAGE and stained with Biosafe Coomassie Stain G-250 (Bio-Rad). Positive fractions were collected and desalted using a PD-10 column (GE Healthcare) using final buffer (50 mm Tris- $\mathrm{HCl}, \mathrm{pH} 8,300 \mathrm{~mm} \mathrm{NaCl}, 0.5 \%$ Triton X-100, protease inhibitors without EDTA). His-Sytx1A was quantified with the Bio-Rad Protein Assay (Bio-Rad) using BSA as standard. Pure His-Sytx1A was kept at $4^{\circ} \mathrm{C}$.

The cytosolic fragment of DCC (GST-DCCCYT) and MUNC18a (GST-Munc18) cloned in pGEX-KD and pGEX-4T-1 vectors, respectively, were expressed in E. coli Rosetta BL21 (DE3) competent cells after induction with $1 \mathrm{~mm}$ IPTG (isopropyl- $\beta$-D-thiogalactoside). Cells were centrifuged at $6000 \times g$ for $20 \mathrm{~min}$, and the pellet was resuspended in ice-cold phosphate buffer (20 mm), pH 7.5, with $250 \mathrm{~mm} \mathrm{NaCl}$ and 1\% Triton X-100. Cells were disrupted by sonication and centrifuged at $12,000 \times g$ for $30 \mathrm{~min}$. The supernatants were incubated overnight at $4^{\circ} \mathrm{C}$ with glutathione-Sepharose $4 \mathrm{~B}$ beads (GE Healthcare). After incubation, beads were washed three times and kept in the same buffer at $4^{\circ} \mathrm{C}$ before used.

Forebrain lysates and lysates from DCC-transfected HEK293 cells (or untransfected cells) were obtained as above. Forebrain protein extracts ( 2 $\mathrm{mg}$ of total protein) were mixed with $500 \mu \mathrm{g}$ of His-Sytx1A. The mixture was incubated on a rocking platform at $4^{\circ} \mathrm{C}$ for $14 \mathrm{~h}$. Then, $200 \mu \mathrm{l}$ of Chelating Sepharose Fast Flow bead suspension (GE Healthcare) was equilibrated with $2 \mathrm{vol}$ of $50 \mathrm{~mm} \mathrm{NiSO}_{4}$ and $2 \mathrm{vol}$ of binding buffer ( 50 $\mathrm{mm}$ Tris- $\mathrm{HCl}, \mathrm{pH} 8,300 \mathrm{~mm} \mathrm{NaCl}, 5 \mathrm{~mm}$ imidazole, $0.5 \%$ Triton X-100, protease inhibitors without EDTA). Equilibrated beads were added to the mixture and incubated at $4^{\circ} \mathrm{C}$ for $30 \mathrm{~min}$. Protein-bead complexes were centrifuged at $2000 \times g$ for $30 \mathrm{~s}$, the supernatant was removed, and the pellet was washed twice with binding buffer and twice with washing buffer (binding buffer plus $50 \mathrm{~mm}$ imidazole). Bound proteins were released by addition of $50 \mu \mathrm{l}$ of binding buffer containing $500 \mathrm{~mm}$ imidazole, incubation for $2 \mathrm{~min}$ on ice, and centrifugation at $2000 \times g$ for $1 \mathrm{~min}$ to remove the beads. The supernatant was collected and resolved on a $7 \%$
SDS-PAGE, transferred onto a nitrocellulose membrane, and detected with $\alpha$-DCC antibody (Oncogene). As a positive control for Sytx1Ainteracting proteins, the membranes were incubated with $\alpha$-SNAP25 antibodies (data not shown) (Synaptic Systems). The same protocol was used for HEK293 cells transfected with DCC or for untransfected cells. To remove EDTA and DTT that could interfere with the pull down, the extracts were washed four times using a Microcon device YM-10, MWCO 10,000 Da (Millipore). Extracts (100 $\mu$ l) were incubated with $500 \mu \mathrm{g}$ of His-Sytx1A as previously described. Controls were performed by incubation with $500 \mu \mathrm{g}$ of HC-TeTx or POP, or without adding recombinant proteins.

In other experiments, rat brains from $\mathrm{P} 1$ animals were homogenized in ice-cold $20 \mathrm{~mm}$ HEPES/KOH, pH 7.2, $120 \mathrm{~mm}$ potassium glutamate, $20 \mathrm{~mm}$ potassium acetate with $1 \%$ Triton $\mathrm{X}-100,10 \mu \mathrm{g} / \mathrm{ml}$ leupeptin, and $10 \mu \mathrm{g} / \mathrm{ml}$ aprotinin and centrifuged at 20,000 $\times g$ for $15 \mathrm{~min}$. Supernatants were incubated with Talon metal affinity resin beads (BD Biosciences) coupled or not to His $_{6}$-Syntaxin 1A-full length, Syntaxin 1A-H3-TM fragment, or with gluthathione-Sepharose beads coupled to GST-GFP, GSTMunc18a, or GST-DCCcyt. After an overnight incubation at $4^{\circ} \mathrm{C}$, the beads were washed three times with homogenization buffer. After adding SDSPAGE sample buffer to the pellets, samples were boiled for $5 \mathrm{~min}$, processed for SDS-PAGE, and transferred to nitrocellulose membranes. Western blot was performed using a monoclonal antibodies against DCC (BD Biosciences Pharmingen) and HRP-coupled rabbit anti-mouse as secondary antibody. Membranes were developed using the ECL method.

In other experiments, P0 brain extracts were incubated with $\mathrm{Ni}^{2+}$ affinity resin beads (BD Biosciences) coupled or not to His-Sytx1A protein. After an overnight incubation at $4^{\circ} \mathrm{C}$, the beads were washed three times with homogenization buffer. After adding SDS-PAGE sample buffer to the pellets, samples were boiled for $5 \mathrm{~min}$, processed for SDSPAGE, and transferred to nitrocellulose membranes. WB was performed using a monoclonal antibody against DCC as primary antibody. Membranes were developed using the ECL method. For the in vitro transcription and translation of Sytx1A using reticulocyte extracts, $\left[{ }^{35} \mathrm{~S}\right]$ methionine-labeled Sytx1A was obtained using the TNT kit (Promega). Two to $4 \mu \mathrm{l}$ of $\left[{ }^{35} \mathrm{~S}\right]$ methionine-labeled Sytx1A were used for pull-down assays, which were performed essentially as described above, except that GST-DCCCYT coupled to glutathione-Sepharose $4 \mathrm{~B}$ beads (GE Healthcare) was used, and that after SDS-PAGE, gels were stained with Coomassie Blue R250, destained, dried, and exposed to a storage phosphor screen (GE Healthcare). Screens were scanned and analyzed with a phosphorimager (Bio-Rad). For competition experiments, nonlabeled His-Sytx1A $(2-4 \mu \mathrm{g})$, purified as above, was added together with the $\left[{ }^{35} \mathrm{~S}\right]$ methionine-labeled Sytx1A.

Surface plasmon resonance analyses. The interaction between DCC and Sytx1A was analyzed using the BIAcore technology. This approach is based on changes in surface plasmon resonance (SPR) signals from chips coated with carboxymethylated dextran to which the proteins or peptides can be covalently cross-linked (Johnsson et al., 1991; Fivash et al., 1998). The putative ligands were flowed at a range of concentrations into the chamber containing the chips, and signals proportional to changes in the refractive index due to increased protein concentration were recorded. In this study, we used a BIAcore T1000 apparatus (Biacore) and CM5 sensor chips. The buffer used for continuous flow was HBS-EP (10 mM HEPES, $\mathrm{pH}$ 7.4, $150 \mathrm{~mm} \mathrm{NaCl}, 3.0 \mathrm{~mm}$ EDTA, 0.05\% surfactant P20). GSTDCCCYT fragment (or control GST protein) was immobilized by activation of the CM5 sensor chip with $50 \mathrm{~mm} N$-hydroxysuccinimide and $200 \mathrm{~mm} N$-ethyl- $N^{\prime}$-(3-diethylaminopropyl)-carbodiimide for $7 \mathrm{~min}$ at a flow rate of $5 \mathrm{ml} / \mathrm{min}$. The protein was injected at a concentration of 1 $\mathrm{mg} / \mathrm{ml}$ in $50 \mathrm{~mm}$ HEPES, $\mathrm{pH} 7.5$, for $15 \mathrm{~min}$, and finally the surface was deactivated by injection of $1 \mathrm{~m}$ ethanolamine, $\mathrm{pH} 8.5$, for $7 \mathrm{~min}$. Purified His-Sytx1A, resuspended in $50 \mathrm{~mm}$ HEPES, pH 7.4, and $300 \mathrm{~mm} \mathrm{NaCl}$, was injected at a range of concentrations (10 nM to $30 \mu \mathrm{M})$ into the previously immobilized GST-DCCCYT fragment. The sensorgrams were analyzed using the BIAevaluation software, version 4.1 (Biacore). The equilibrium dissociation constants $\left(K_{\mathrm{D}}\right.$ values) were calculated either from steady-state binding data or from kinetic parameters $\left(k_{\text {on }}\right.$ and $\left.k_{\text {off }}\right)$. A peptide corresponding to T1 region of DCC (CTRRSSAQQRKKRATH SAGKRKGSQKDLR) was purchased from Invitrogen and immobilized by activation with $50 \mathrm{~mm} N$-hydroxysuccinimide and $200 \mathrm{~mm} N$-ethyl- 
$N^{\prime}$-(3-diethylaminopropyl)-carbodiimide for $8 \mathrm{~min}$ at a flow rate of $5 \mathrm{ml} / \mathrm{min}$, followed by injection of the peptide $(20 \mathrm{~min})$ at a concentration of $1 \mathrm{mg} / \mathrm{ml}$ in 50 mм HEPES, pH 7.5, and by $8 \mathrm{~min}$ of deactivation with $1 \mathrm{M}$ ethanolamine, $\mathrm{pH}$ 8.5. A control peptide corresponding to the P3 region of DCC (EQMASLEGLM KQLNA) was used as a negative control. Purified His-Sytx1A was injected at a range of concentrations ( $10 \mathrm{~nm}$ to $30 \mu \mathrm{M}$ ), and the binding data were analyzed as described above.

$B O D I P Y$ staining. For staining membrane vesicles, hippocampal explants were cultured as above and labeled with BODIPY ceramide (Invitrogen) for $30 \mathrm{~min}$ at room temperature and then chased for $2.5-3 \mathrm{~h}$ at $37^{\circ} \mathrm{C}$ (Pagano et al., 1991; Paglini et al., 2001). Explants were then treated with Netrin-1-containing or control media for 15,30 , or $60 \mathrm{~min}$. Cultures were also treated with $25 \mathrm{~nm}$ BoNT/A, 15 nм BoNT/ $\mathrm{C} 1$, or $2 \mathrm{~nm}$ TeNT, $2-3 \mathrm{~h}$ before the addition of Netrin-1. Explants were fixed for $1 \mathrm{~h}$ at $4^{\circ} \mathrm{C}$ with $2 \%(\mathrm{w} / \mathrm{v})$ paraformaldehyde in PBS, washed with PBS, and mounted with Mowiol. Confocal images of axonal growth cones were acquired using a TCS SP2 confocal microscope. Data were collected from at least three independent experiments, and an average of 40 growth cones for each condition was analyzed for BODIPY staining.

Immunohistochemistry. Hippocampal explants were immunostained with an antibody against neuron-specific class $\beta$ III-tubulin (clone TUJ-1; 1:4000). Explants were incubated with a biotinylated secondary antibody and streptavidin-HRP complex, followed by reaction with diaminobenzimidine and $\mathrm{H}_{2} \mathrm{O}_{2}$, or processed for immunofluorescence. Hippocampal primary cultures were stained with mouse $\alpha$-DCC (1: 500; Oncogene), $\alpha$-Sytx1 (1:1000), $\alpha$-SNAP25 (1:500), $\alpha$-VAMP2 (1:500), $\alpha$-TI-VAMP (1:500), followed by Alexa 568- and Alexa 488-coupled antibodies.

Quantification of explants. Quantification of explants was as described previously (Yee et al., 1999; Schiavo et al., 2000; Pozas et al., 2001). For hippocampal explants, the density of axons in $50 \mu \mathrm{m}$ stripes was counted in the proximal, distal, and lateral quadrants. The proximal/distal ratio (attraction index) was also calculated (Pozas et al., 2001). Data were collected from at least three independent experiments (three to seven experiments per condition). The number of cultures quantified is shown in the figures.

Quantification of immunofluorescence experiments. Confocal images of axonal growth cones were acquired sequentially to ensure there was no bleed-through of signal between channels, using a TCS SP2 confocal microscope (Leica Microsystems) (63× objective, 1.4 NA). Single section images were analyzed using the built-in Leica confocal software (LCS) multicolor program package. Briefly, a scatter plot of pixel intensities (1-225) of the two channels was built and a region extending along the midline $(X=Y)$ of the plot was considered as "yellow" (overlap between the two channels). Pixel intensities $<40$ were consid-
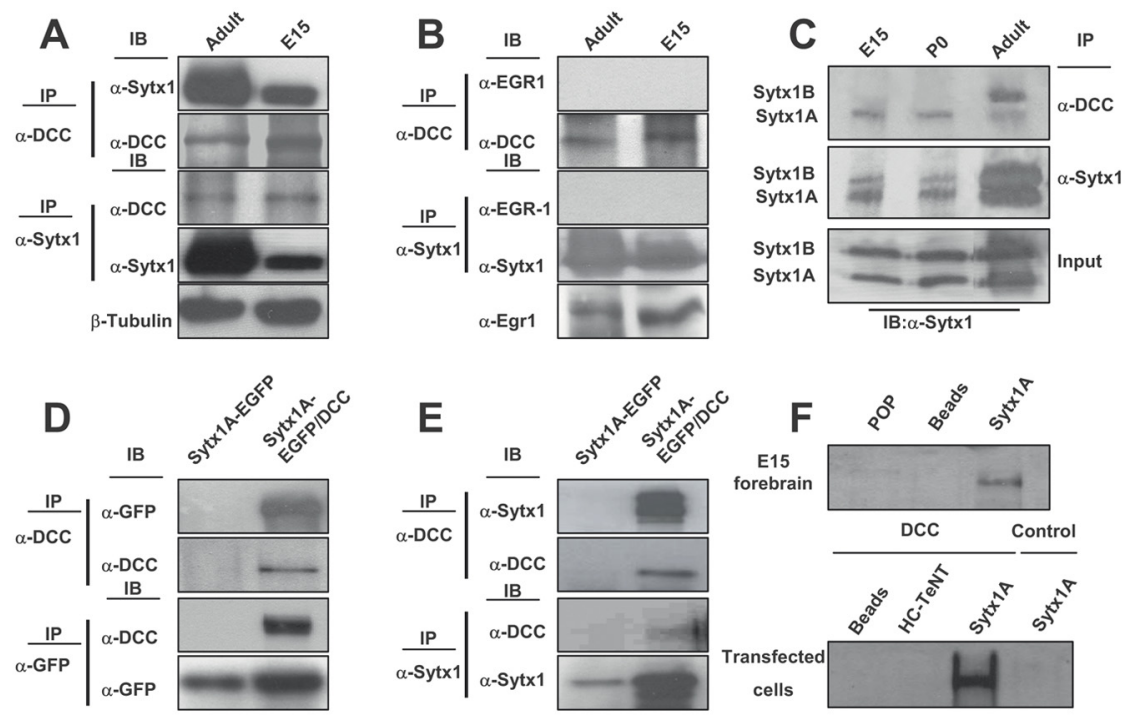

G

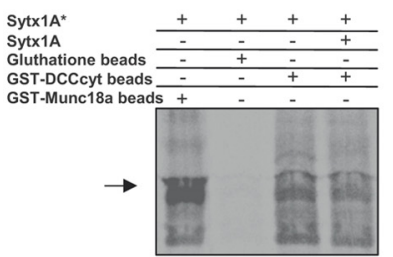

H

I
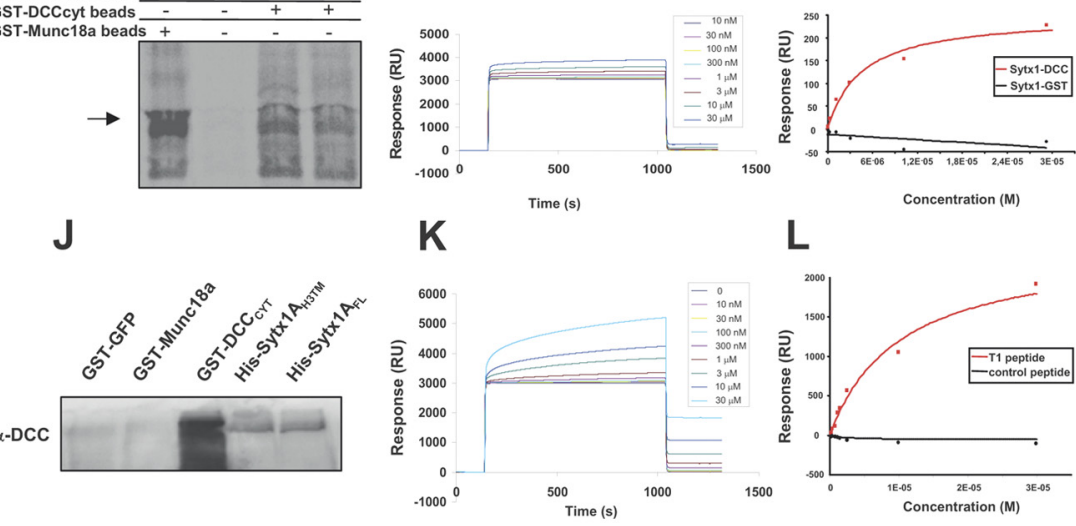

L

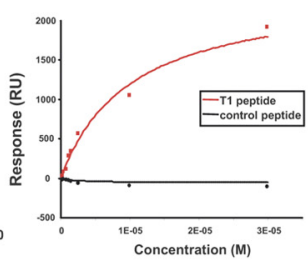

Figure 1. Sytx 1 coassociates with the $D C C$ receptor in brain tissue and in transfected cells. $A, D C C$ and Sytx 1 immunoprecipitation of 15 and adult forebrain homogenates. DCC immunoprecipitation yields coimmunoprecipitation of Sytx 1 in both samples (top panels). Sytx1 immunoprecipitation yields coassociation of DCC (bottom panels). Anti- $\beta$ III-tubulin antibodies were used as loading controls. B, DCC and Sytx1 immunoprecipitation assays do not result in coimmunoprecipitation of Egr1. Levels of Egr1 protein lysates are shown (bottom).C, DCC associates with $A$ and $B$ isoforms of Sytx1. Forebrain homogenates were immunoprecipitated and immunocomplexes were subjected to urea/SDS-PAGE. DCC coimmunoprecipitates two Sytx1 bands corresponding to Sytx1A and 1B (top panel). D, E, Coimmunoprecipitation experiments in HEK293 cells. DCC immunoprecipitation results in coassociation with Sytx1A, visualized either with anti-GFP (D) or antiSytx1 $(\boldsymbol{E})$ antibodies. The reverse immunoprecipitation with anti-GFP or anti-Sytx1 antibodies also reveals DCC $(\boldsymbol{D}, \boldsymbol{E})$. Note that the efficiency of the immunoprecipitation with anti-GFP antibodies is consistently higher than when using anti-DCC antibodies, which yields low recovery of proteins in this condition. $F, D C C$ affinity pull-down experiments with purified His-Sytx1A. Incubation with anti-DCC antibody reveals DCC in the beads coupled to His-Sytx1A, but not in control beads. G, Sytx1A was transcribed and translated in vitro in the presence of $\left[{ }^{35}\right.$ S]Met, and incubated with glutathione-Sepharose beads coupled to GST-DCC ${ }_{\mathrm{CYT}}$ or GST-MUNC18a. After SDS-PAGE, gels were exposed to a storage phosphor screen. [ $\left.{ }^{35} \mathrm{~S}\right] \mathrm{Met}-\mathrm{Sytx} 1 \mathrm{~A}$ binds to $\mathrm{DCC}$ (YT (as well as to MUNC18a), but not to empty beads. Binding of $\left[{ }^{35}\right.$ S]Met-Sytx1A to DCC $C_{C Y}$ is decreased in the presence of nonradioactive Sytx $1 A$ (Sytx $\left.1 A^{*}\right)$. $\boldsymbol{H}$, Sensorgram showing binding between the GST-DCC ${ }_{\text {CYT }}$ domain and His-Sytx1A. Increasing concentrations of His-Sytx1A were injected into a chip where the GST-DCC CYT was crosslinked; the interaction was recorded as SPR changes [in response units (RU)]. Increasing concentrations of His-Sytx1A yielded higher responses. I, Plot of the steady-state response (in RU) between GST-DCC $\mathrm{CYT}_{\mathrm{T}}$ and a range of concentrations of His-Sytx1A (red) or between control GST and His-Sytx1A (black). No binding is detected when GST protein was cross-linked, whereas a saturable response is observed when GST-DCC $\mathrm{CYT}_{\mathrm{T}}$ was immobilized.J, Pull-down experiments in which brain extracts (PO) were passed through $\mathrm{Ni}^{2+}$-affinity columns to which recombinant His-Sytx1A or His-Sytx1AH3TM proteins were coupled. Western blot analyses show coprecipitation of DCC with similar efficiencies in both cases. No coprecipitation of DCC is detected when extracts are incubated with control GST-GFP or GST-MUNC18a columns, whereas strong coprecipitation was observed with GST-DCCcyt. The high DCC signal in GST-DCCcyt samples is probably due to multimerization of the DCC receptor through the P3 domain. $\boldsymbol{K}$, Sensorgram showing binding between the T1DCC peptide and purified His-Sytx1A. The peptide was immobilized as described in Material and Methods, and then increasing concentrations of His-Sytx1A were injected. Responses (in RU) increase at increasing concentrations of His-Sytx1A as a function of the time (in seconds). $L$, Plot of the steadystate response between $\mathrm{T} 1$ peptide and His-Sytx1A (red) or between a control peptide and His-Sytx $1 \mathrm{~A}$ (black). While no binding is detected when the control peptide is cross-linked, specific binding is observed when T1 peptide is immobilized. 

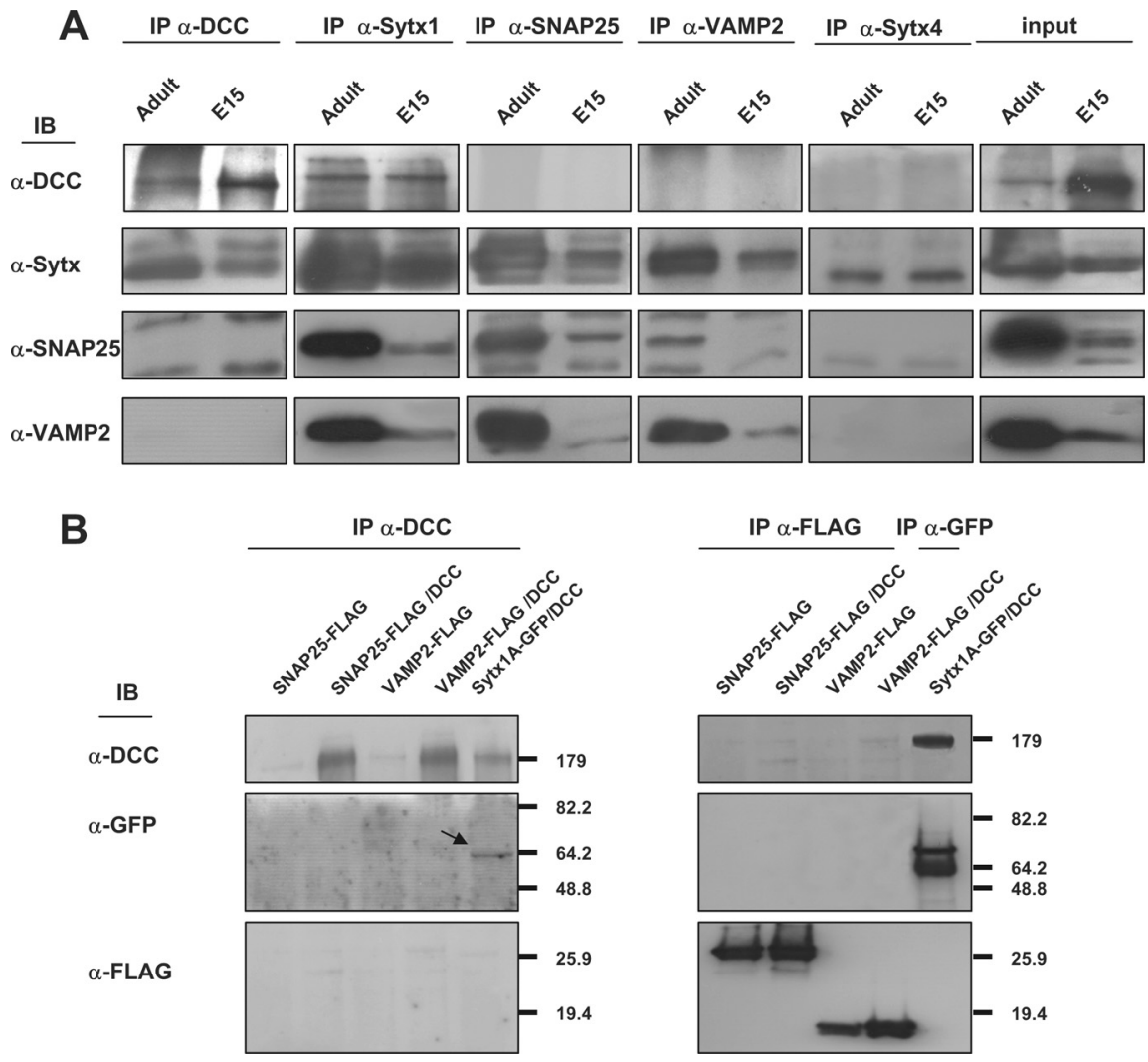

Figure 2. The DCC receptor does not coassociate with SNAP25, VAMP2, or Syntaxin 4. A, Coimmunoprecipitation experiments in brain lysates (E15 and adult). Whereas DCC and Sytx 1 coimmunoprecipitate, the DCC receptor does not coassociate with SNAP25, VAMP2, or Sytx 4 in brain lysates. The Sytx antibody used for the immunoblot was an anti-Sytx1A antibody in all cases, except in the column immunoprecipitated with anti-Sytx 4 antibodies. Inputs are shown to the right. $B$, Coimmunoprecipitation experiments in HEK293 cells transfected with pCMVDCC and SNAP25-FLAG, VAMP2-FLAG or Sytx1A-EGFP DNAs. DCC immunoprecipitation results in coassociation with Sytx1A visualized with anti-GFP antibodies, but not with SNAP25 or VAMP2 (FLAG antibodies). The reverse immunoprecipitation with anti-FLAG antibodies did not reveal DCC coimmunoprecipitation.

ered as background and omitted from the study. Regions of interest (growth cones) were drawn on the images, and overlapped pixels were divided by the area of these regions of interest. Colocalization values were normalized to the intensity levels of red (DCC) and green (Sytx1A) channels, to refer colocalization to total protein content. We quantified DCC/ Sytx1 colocalization in the entire growth. Similar experiments were performed with SNAP25 and VAMP2 antibodies. For the calculation of membrane/growth cone ratios in DCC immunofluorescence, the signal intensity at the surface of growth cones (the outermost signals, defined as a region comprised from the outline up to $400 \mathrm{~nm}$ inside the growth cone) were referred to the signals detected inside the growth cones. At least five separate experiments (usually seven to eight) were performed for each condition. The number of axonal growth cones counted is shown in the figures.

In vivo analysis of commissural axon pathfinding. The analysis of commissural axon trajectories was performed as described previously (Stoeckli and Landmesser, 1995; Fazeli et al., 1997). In brief, fertilized eggs were windowed on the third day of incubation. Extra-embryonic membranes were removed to access the spinal cord in ovo. The plasmids pIRESEGFP, Sytx1AH3TMpIRESEGFP, or those encoding other fusion proteins (Sytx1AH3pIRESEGFP, Sytx1ATMpIRESEGFP) under the control of the $\beta$-actin promoter in PBS $(100 \mathrm{ng} / \mu \mathrm{l})$ were injected into the central canal using glass capillaries. For visualization and control of injection quality and quantity, $0.04 \%$ trypan blue was added. For electroporation, we used platinum electrodes connected to a BTX square wave electroporator. Electrodes were positioned parallel to the longitudinal axis of the lumbosacral spinal cord of the chicken embryo, as detailed previously (Perrin and Stoeckli, 2000; Bourikas et al., 2005). Five pulses of $26 \mathrm{~V}$ and $50 \mathrm{~ms}$ duration with a $1 \mathrm{~s}$ interpulse interval were applied.
The same parameters were used for the transfection of dsRNA derived from Sytx1A, TIVAMP, or neuron-glia cell adhesion molecule (NgCAM). dsRNA was produced by in vitro transcription as described previously (Pekarik et al., 2003). For the production of dsRNA derived from Sytx1A, NgCAM, or TI-VAMP, we used ESTs obtained from Geneservices (Bourikas et al., 2005). dsRNA was injected in PBS $(250 \mathrm{ng} / \mu \mathrm{l})$ and $0.04 \%$ trypan blue. After electroporation, eggs were sealed with a glass coverslip and melted paraffin or Scotch tape and incubated for another $2 \mathrm{~d}$.

Embryos were killed between stages 25 and 26 (Hamburger and Hamilton, 1992). The spinal cord was removed from the embryo, opened at the roof plate ("open-book" preparation), and fixed in $4 \%$ paraformaldehyde for $30-60 \mathrm{~min}$.

Alternatively, 250- $\mu \mathrm{m}$-thick transverse slices were cut from fixed embryos. In both cases, the trajectories of commissural axons at the lumbosacral level of the spinal cord were visualized by the application of the lipophilic dye Fast DiI (dissolved at $5 \mathrm{mg} / \mathrm{ml}$ in methanol; Invitrogen) to the cell bodies. Care was taken to exclusively label the dorsal-most population of commissural neurons to avoid confusion with more ventral populations that have distinct pathfinding behavior. For the quantification of the axonal pathfinding phenotype (see Figs. $8 K$, $9 K$ ), we used open-book preparations. After visualizing a subset of dorsal commissural axons by dye tracing, we assessed the percentage of fibers that extended from the dorsal spinal cord but that failed to enter and cross the floor plate. We classified the phenotype as weak when between 20 and $50 \%$ of the fibers failed to reach the contralateral floor plate border, and as strong when $50 \%$ or more fibers were affected. It is important to emphasize that the proportion of cells that are successfully transfected by this in ovo electroporation procedure is on average 60\% (Perrin and Stoeckli, 2000). Therefore, $\sim 40 \%$ of the cells are expected to be wild type and to cross the floor plate normally.

Statistics. Data were expressed as means \pm SEM and were analyzed statistically using ANOVA (Statgraphics Plus 5.1).

\section{Results}

The DCC receptor associates with the t-SNAREs Sytx1A/1B

Axonal guidance receptor function is modulated by interaction with other membrane proteins, including guidance receptors (Hong et al., 1999), and SNARE protein functions and exocytosis essentially operate through complex and multiple protein interactions (see above) (Verhage et al., 2000; Castillo et al., 2002; Jahn and Scheller, 2006; de Wit et al., 2009; Südhof and Rothman, 2009). We therefore hypothesized that there is a cis-direct interaction between the Netrin-1 receptor DCC and the plasmalemma t-SNAREs SNAP25 and Sytx1 and that this interaction might influence exocytosis. In preliminary experiments on brain lysates, DCC pull-down assays coimmunoprecipitated Sytx1 (Fig. 1). In contrast, coimmunoprecipitation assays on brain lysates or transfected cells revealed that DCC does not interact with the SNAREs VAMP2 and SNAP25, or with Sytx4, a SNARE involved in protein traffic (Fig. 2). This observation suggested that the canonical Sytx1 SNARE partners (SNAP25 and VAMP2) were 
not associated with the DCC-Syntaxin 1 complexes (see also below).

We thus focused on the association of DCC and Sytx1. DCC antibodies immunoprecipitated in brain lysates a band of $35 \mathrm{kDa}$, which corresponded to SytxlA and $1 \mathrm{~B}$ isoforms (WB with the HPC-1 Mab; Fig. 1A) (Ruiz-Montasell et al., 1996). A $180 \mathrm{kDa}$ band corresponding to DCC was identified after immunoprecipitation with anti-Sytxl antibodies (Fig. 1A). When DCC or Sytx1 immunoprecipitates were revealed with control antibodies (Fig. $1 B$ ), no signal was detected. Furthermore, immunoprecipitation with beads alone or with irrelevant antibodies (anti-GFP or anti-Egr1) did not reveal coassociation (data not shown). Because Sytx1A and $1 \mathrm{~B}$ are difficult to resolve in conventional SDS-PAGE, immunoprecipitates were run in urea/ SDS-PAGE. These experiments showed that Sytx1A and 1B isoforms coimmunoprecipitated with DCC, but with distinct developmental profiles (Fig. 1C). Together, these results indicate an in vivo association of Sytx1A/1B and DCC in the developing and adult brain.

To corroborate the interaction of DCC and Sytx1A, we performed several experiments. First, in coimmunoprecipitation assays in HEK293 or COS- 1 cells transfected with constructs of both proteins (pRcCMVDCC or SytxlAEGFP), Sytx1AEGFP was detected by WB in lysates immunoprecipitated with anti-DCC antibodies. Pull-down assays with anti-GFP or anti-Sytxl antibodies revealed DCC protein. No coimmunoprecipitation was detected when cells were transfected with either Sytx1AEGFP or DCC DNA alone (Fig. $1 D, E$ ). These data indicate that Sytx1A and DCC coassociate after expression in non-neuronal cells. Second, affinity pull-down experiments with purified His-Sytx1A were performed. Bacterially expressed His-Sytx1A or control His-tagged proteins (POP; HC-TeTx) were purified and incubated with either forebrain lysates or extracts from HEK293 cells transfected with DCC. Protein complexes were eluted and fractions were labeled with $\alpha$-DCC antibodies. DCC was present in the samples incubated with Sytx1A-FL, both in extracts from brain tissue and in DCC-transfected cells, but not in beads coupled to control proteins (Fig. $1 F$ ).

To provide evidence of direct binding of Sytx1A to DCC, Sytx1A was transcribed and translated in vitro using reticulocyte lysates, in the presence of $\left[{ }^{35} \mathrm{~S}\right] \mathrm{Met}$, and incubated with glutathione-Sepharose beads coupled to GST-DCC CYT $_{\text {, GST- }}$ MUNC18a, or control beads (Fig. 1G). Bound proteins were eluted and revealed by autoradiography. The results showed binding of

IB $\alpha$-DCC

IB $\alpha$-GFP

D top right panel.
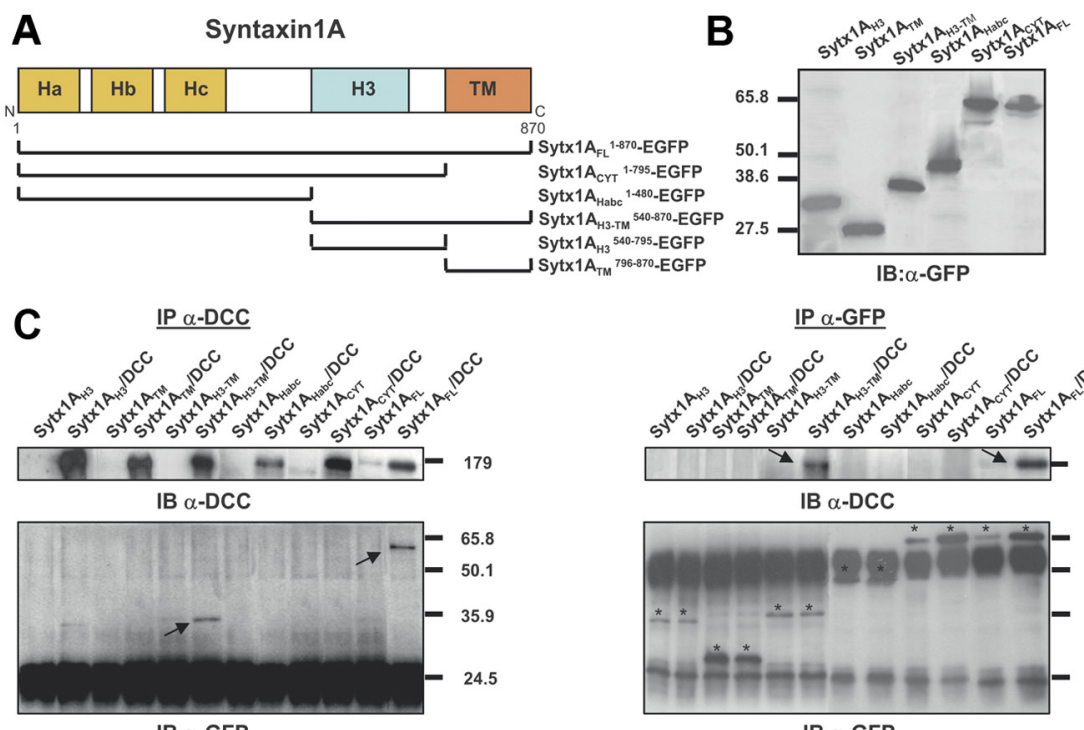

DCC

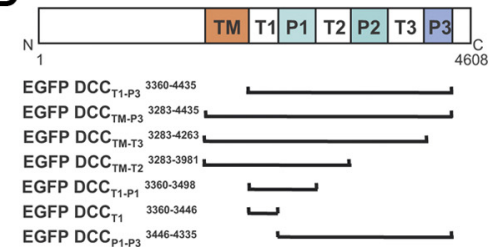

$\underline{\text { IP } \alpha \text {-GFP }}$
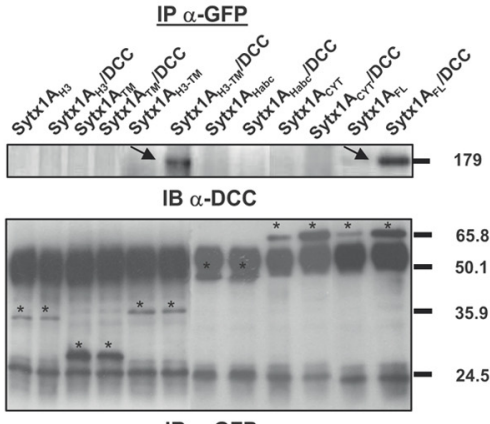

IB $\alpha$-GFP

$\mathbf{E}$

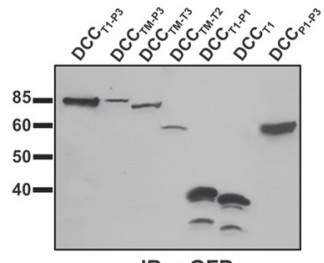

IB $\alpha$-GFP
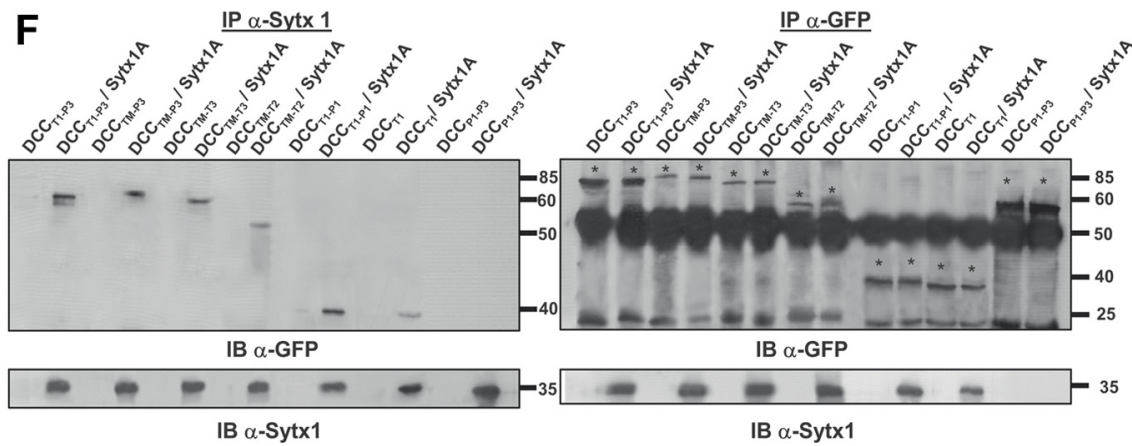

Figure 3. Characterization of protein regions required for Sytx $1 A / D C C$ interaction. $A$, Diagram summarizing Sytx $1 A$ domains and the truncated Sytx1AEGFP chimeras generated. $\boldsymbol{B}$, Expression of the several Sytx1AEGFP DNAs in HEK293 cells, showing that they generate proteins of the appropriate $M_{\mathrm{r}}$ between 65 and $27 \mathrm{kDa}$, as revealed by immunoblotting with anti-GFP antibodies. $C$, Coimmunoprecipitation experiments in HEK293 cells cotransfected with the distinct Sytx1AEGFP constructs together with pCMV or pCMVDCC. DCC immunoprecipitations (200 $\mu \mathrm{g}$ ) were revealed by immunoblotting with anti-DCC or anti-GFP antibodies (left panel). Cells cotransfected with $p C M V D C C$ and Sytx $1 A_{\mathrm{FL}}$ EGFP or Sytx $1 \mathrm{~A}_{\mathrm{H} 3 \mathrm{TM}}$ EGFP show positive coimmunoprecipitation of Sytx $1 \mathrm{~A}$ fusions. The reverse immunoprecipitation assays with anti-DCC antibodies also show coimmunoprecipitation with DCC, exclusively when cells are cotransfected with Sytx $1 A_{\mathrm{FL}}$ EGFP or Sytx $1 \mathrm{~A}_{\mathrm{HзTM}}$ EGFP DNAs (arrows in top right panel). The bands corresponding to the distinct Sytx1AEGFP chimeras are labeled with asterisks in the bottom right panel label. D, Diagram summarizing the DCC domains and the truncated EGFPDCC chimeras generated. $E$, Western blot, revealed with an anti-GFP antibody, demonstrating appropriate $M_{\mathrm{r}}$ (between 90 and $40 \mathrm{kDa}$ ) of the distinct EGFPDCC chimeras expressed in HEK293 cells. $\boldsymbol{F}$, Coimmunoprecipitation experiments in HEK293 cells cotransfected with the distinct EGFPDCC constructs together with pRcCMV or pRcCMVSytx1A DNAs. Sytx 1 immunoprecipitations $(200 \mu \mathrm{g})$ were revealed by immunoblotting with anti-GFP or anti-Sytx1 antibodies (left panel). All the cells cotransfected with pRCCMVSytx1A and the EGFPDCC constructs show coimmunoprecipitation of EGFP-tagged DCC chimeras (arrows), except when cells are cotransfected with EGFP $_{\mathrm{P}_{1} \mathrm{P}_{3}} \mathrm{DCC}$ DNA. The reverse immunoprecipitation assays with anti-GFP antibodies also reveal coimmunoprecipitation with Sytx $1 \mathrm{~A}$ (arrows in bottom right panel) in all cotransfected cells, except in those transfected with EGFP $P_{P 1-P 3} D C C D N A$. The bands corresponding to the distinct Sytx1AEGFP chimeras are labeled with asterisks in the

$\left[{ }^{35} \mathrm{~S}\right]$ Met-Sytx1A to DCC $_{\mathrm{CYT}}$ (as well as to MUNC18a), but not to empty beads. Furthermore, the binding of $\left[{ }^{35} \mathrm{~S}\right]$ Met-Sytx1A to DC$\mathrm{C}_{\mathrm{CYT}}$ was decreased in the presence of nonradioactive Sytx1A (Fig. $1 G)$. To further demonstrate the direct interaction between Sytx1A and DCC, we applied the BIAcore technique, in which purified GST- 
A

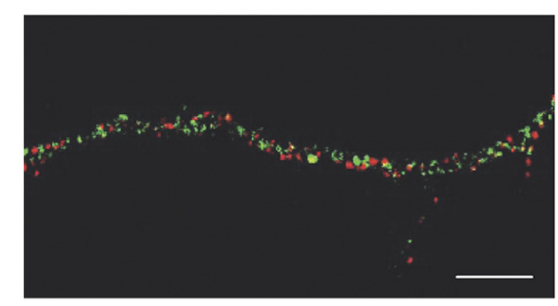

N15

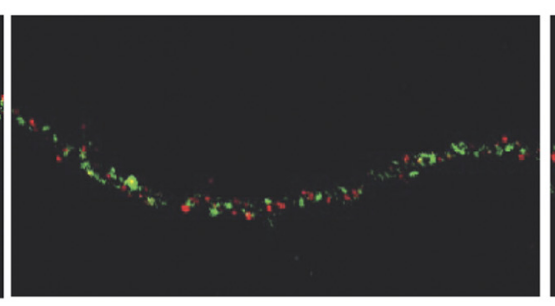

N30'

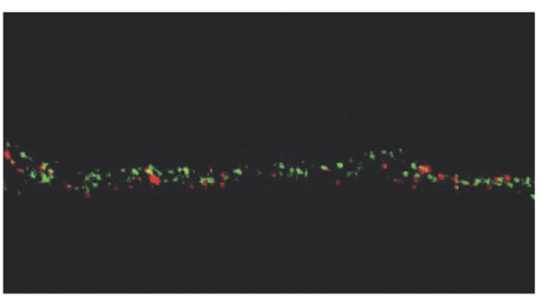

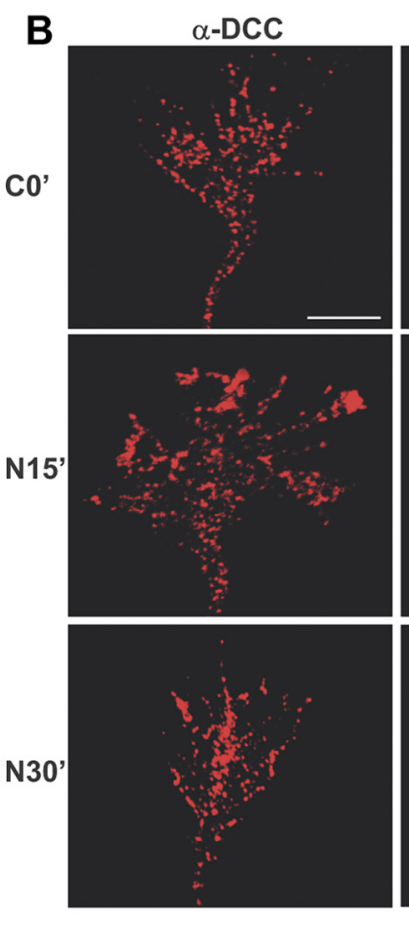

E
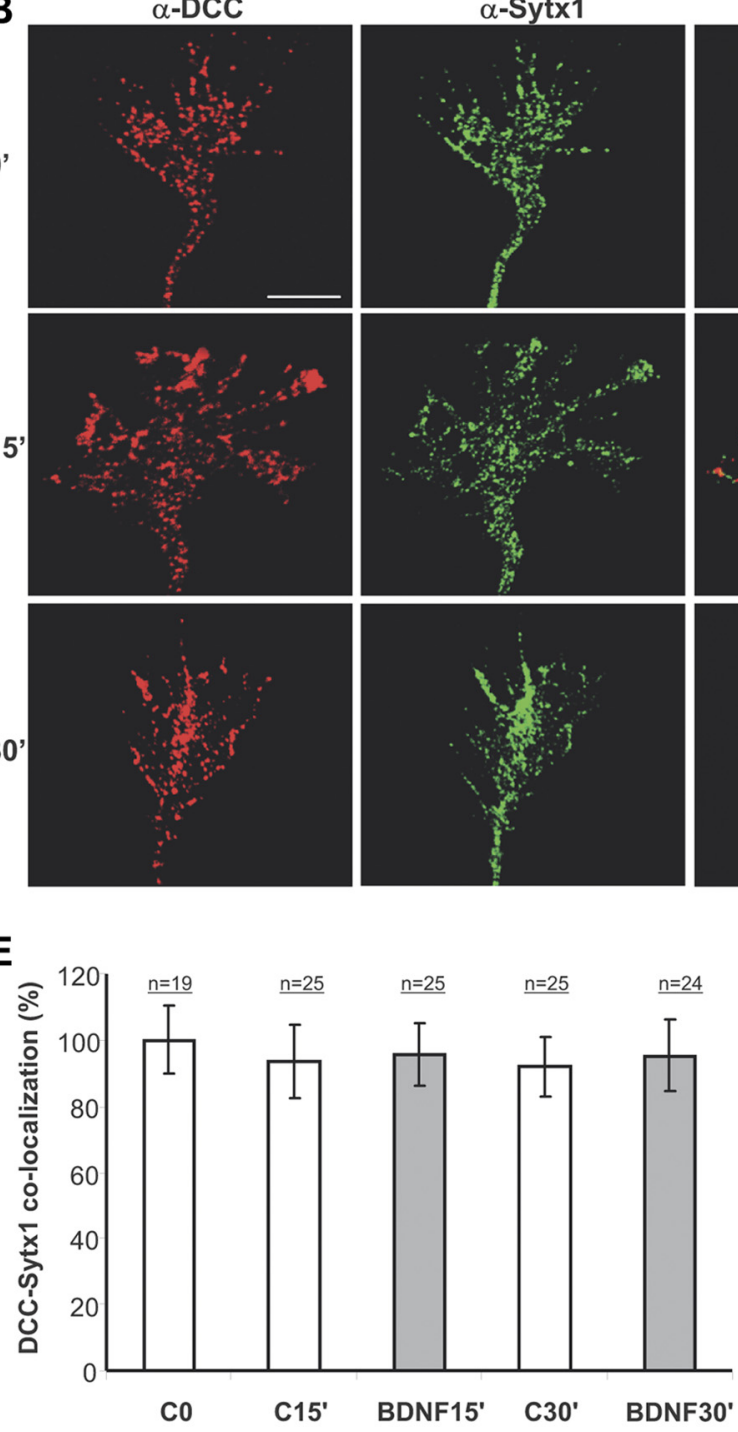

Merge
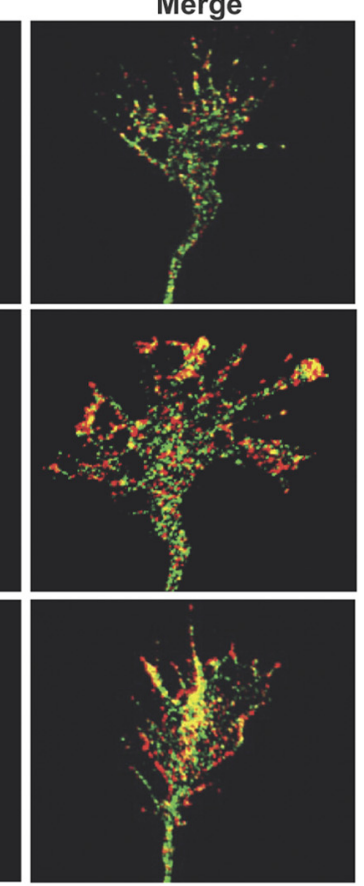

C
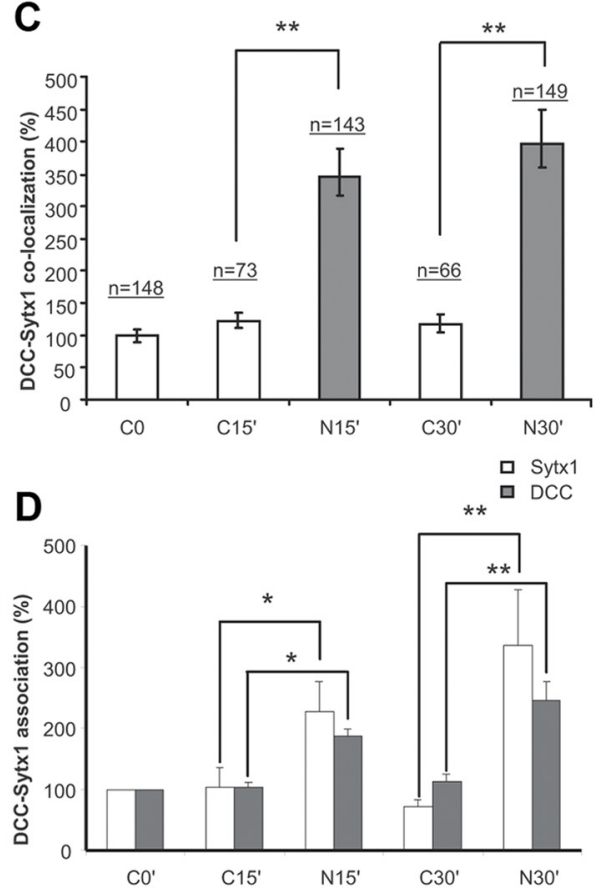

$\mathbf{F}$

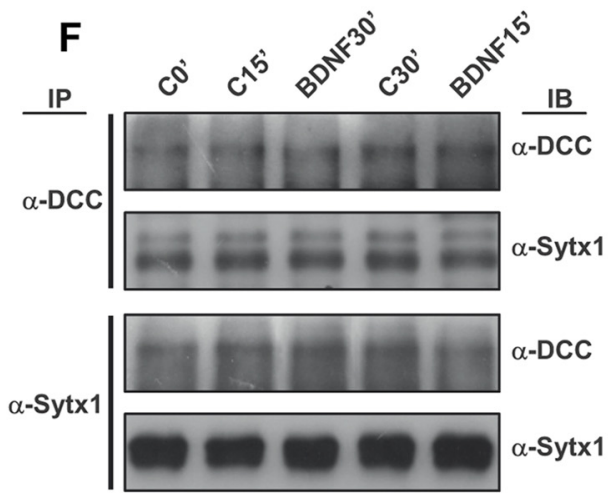

G
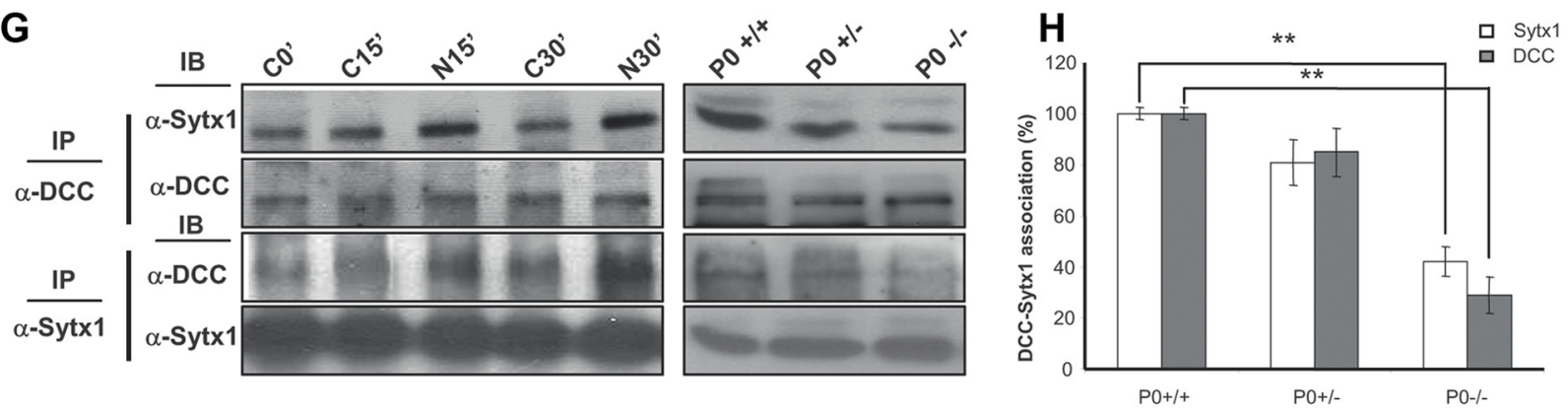

Figure 4. Netrin-1, but not BDNF, triggers DCC mobilization and DCC/Sytx 1 colocalization. $A$, Confocal images of hippocampal axonal shafts treated with Netrin-1-conditioned media for 0,15 , and 30 min, immunolabeled for DCC (red) and Sytx1A (green). Note that DCC and Sytx1A do not colocalize in axonal shafts in either condition. $\boldsymbol{B}$, Confocal images of (Figure legend continues.) 
DCC $_{\text {CYT }}$ protein was immobilized on a CM5 sensor chip by EDC/ NHS coupling. Solutions containing different concentrations of purified His-Sytx1A (10 nM to $30 \mu \mathrm{M}$ ) were passed through the chamber containing the chip, and binding was analyzed according to the SPR signal obtained in real time. We found a specific interaction between the two proteins, which was not detected when control GST protein was immobilized or when an empty chip was used (Fig. $1 H, I)$. Quantitative evaluation of five independent experiments showed that the two molecules interacted with a $K_{\mathrm{D}}$ of $\sim 2.4 \pm 0.7$ $10^{-6} \mathrm{M}$ (Fig. $\left.1 H, I\right)\left(k_{\text {on }}, 1.8 \times 10^{4} \mathrm{M}^{-1} \mathrm{~s}^{1} ; k_{\text {off }}, 0.033 \mathrm{~s}^{-1}\right)$. Together, these biochemical and biophysical experiments show that recombinant DCC and Sytx1 interact directly in vitro.

\section{The transmembrane- $\mathrm{H} 3$ region of Sytx1A and the $\mathrm{T} 1$ region of DCC are required for Sytx1A/DCC interaction}

We next attempted to identify the domains of Sytx 1 required for its interaction with DCC. Sytx1A contains a transmembrane domain (TM) and a cytosolic tail containing the H3 (SNARE) motif and the N-terminal Habc domain (Fig. 3A) (Sutton et al., 1998; Dulubova et al., 1999). While the H3 domain interacts with other SNARE proteins, the Habc region modulates the formation of the SNARE complex (Misura et al., 2000). We generated several truncated Sytx1A constructs to which EGFP was fused at the $\mathrm{N}$-terminal region (Fig. $3 B$ ). Constructs were transfected in combination with DCC in HEK293 cells, and lysates were processed for immunoprecipitation. DCC immunoprecipitation yielded coimmunoprecipitation of EGFP-tagged full-length Sytx1A (Fig. $3 C$, left panel). Cotransfection with either the Sytx1ACYTEGFP or the Sytx1AHabc EGFP constructs did not reveal coassociation. In contrast, DCC antibodies coimmunoprecipitated the Sytx1AH3TMEGFP chimera (Fig. 3C, arrows), but no coimmunoprecipitation was detected after transfection with DNAs encoding for Sytx1ATM EGFP protein. Transfection with Sytx1AH3EGFP yielded a very weak coassociation (Fig. 3C, left panel). The reverse experiments, using $\alpha$-GFP antibodies to immunoprecipitate EGFP-tagged Sytx1A chimeras, and immuno-

\footnotetext{
$\leftarrow$

(Figure legend continued.) hippocampal growth cones treated with Netrin-1-conditioned media for 0-30 min, immunolabeled for DCC and Sytx1. Note increased DCC/Sytx1 colocalization in growth cones incubated with Netrin-1. C, Quantification of DCC/Sytx1 colocalization signals in hippocampal growth cones (expressed as percentage of DCC/Sytx1 colocalization over total Sytx1 signals) in cultures treated with Netrin-1-conditioned (black bars) or controlconditioned (white bars) media. D, Quantification of DCC/Sytx1 coimmunoprecipitation in hippocampal cultures treated with Netrin-1. Sytx1 immunoprecipitation reveals an increase in coassociated DCC in neurons treated with Netrin-1 (black bars). Immunoprecipitation with anti-DCC antibodies shows a marked increase in Sytx1 signals (white bars). $\boldsymbol{E}$, Quantification of DCC/Sytx1 colocalization signals in hippocampal growth cones (expressed as percentage of DCC/Sytx1 colocalization over total Sytx1 signal) in cultures treated with BDNF (gray bars) or control (white bars). Note that BDNF does not increase colocalization of DCC and Sytx1. F, Western blots from hippocampal cultures treated with BDNF for 15-30 min and immunoprecipitated with anti-DCC or anti-Sytx1A antibodies. Immunoblots reveal that BDNF does not increase the coassociation of DCC with Sytx1A. G, Western blots from hippocampal cultures treated with Netrin-1-conditioned (N) or control-conditioned (C) media for 0-30 min (left), and immunoprecipitated with anti-DCC (top panel) or anti-Sytx1 (bottom panel) antibodies. Immunoblots reveal increased association of DCC and Sytx 1 in neuronal cultures treated with Netrin-1. Immunoprecipitations of brain lysates from newborn wild-type, heterozygous and homozygous netrin-1 mutant mice reveal decreased DCC/Sytx1 association in the null mutants (right). Note decreased coimmunoprecipitation of Sytx1 (top) and DCC (middle) in null-mutant brains. $\boldsymbol{H}$, Quantification of DCC and Sytx 1 coimmunoprecipitations in homogenates from newborn wild-type, heterozygous and homozygous netrin-1 mutant mice. Immunoprecipitations with either anti-DCC (white bars) or anti-Sytx1 (black bars) antibodies reveal a marked reduction in DCC/Sytx1 coassociation in netrin-1-null mutants. Significant differences are labeled by asterisks ( ${ }^{*} p \leq 0.05 ;{ }^{* *} p \leq 0.001$ ). Scale bar: $\boldsymbol{A}, \boldsymbol{B}, 3 \mu \mathrm{m}$. Error bars indicate SEM.
}

blotting with $\alpha$-DCC antibodies, yielded identical results as above (Fig. $3 C$, right panel). Thus, only when cells were cotransfected with DCC and Sytx1AFLEGFP or Sytx1AH3TMEGFP was the DCC protein pulled down (Fig. 3C, arrows). These results reinforce the notion that DCC coassociates with Sytx1A and demonstrates that the H3TM region of Sytx1A is required for the interaction with DCC.

DCC contains an extracellular domain, a single transmembrane domain, and a cytosolic tail with three proline-rich regions (P1, P2 and P3) separated by three tails (regions T1, T2 and T3; Fig. 3D). Because Sytx1A lacks an extracellular domain, and the H3TM region of Sytx1A is required for association with DCC, we hypothesized that Sytx1A and DCC interact through the transmembrane region and/or cytosolic tail of DCC. We generated five DCC chimeras tagged with EGFP at the $\mathrm{N}$ terminus (Fig. 3E): the entire DCC cytosolic tail and transmembrane region (EGFPDCCTM-P3), successively deleted P3-P1 domains (EGFPDCCTM-T3, EGFPDCCTM-T2, EGFPDCCTM-T1), and an EGFPDCCT1-P3 chimera lacking the transmembrane region. These EGFPDCC chimeras expressed proteins of the appropriate size (Fig. 3E) and were cotransfected with Sytx1A. When lysates were pulled down with $\alpha$-Sytx 1 antibodies and the immunocomplexes were probed with $\alpha$-GFP antibodies, Sytx1A interacted with the cytosolic DCC tail both in the presence and in the absence of the TM region (EGFPDCCTM-P3 and EGFPDCCT1-P3 constructs; Fig. $3 F$, left panel). Sytx1A/DCC coimmunoprecipitation was also detected when the $\mathrm{P} 3$ or the $\mathrm{P} 2-\mathrm{P} 3$ domains were deleted, but no coimmunoprecipitation was detected in cells transfected with the EGFPDCCP1-P3 DNA, demonstrating that this region is not required for interaction with Sytx $1 \mathrm{~A}$ (Fig. $3 F$ ). We generated an additional chimera containing the T1 region (EGFPDCCT1). Sytx1A coimmunoprecipitated with EGFPDCCT1-P1 and EGFPDCCT1 proteins, indicating that the T1 region of DCC is sufficient for interaction with Sytx1A (Fig. $3 F$ ). Identical results were obtained in the reverse coimmunoprecipitation experiments (i.e., immunoprecipitation of EGFPDCC chimeras, followed by immunoblotting with the HPC-1 $\alpha$-Sytx1 antibody). Only when the T1 region of DCC was deleted did the DCC/Sytx1A interaction not occur (Fig. 3F, right panel). Our findings indicate that the region of DCC required for its interaction with Sytx1A is the T1 region.

These conclusions were further strengthened by pull-down experiments showing that purified ${ }_{\mathrm{CYT}} \mathrm{DCC}$ recruits truncated Sytx1AH3TM protein (the Sytx1A region responsible for binding to DCC), and by BIAcore experiments, in which an immobilized T1 peptide (the 29 aa DCC region responsible for the interaction) was also found to bind purified Sytx1A (Fig. $1 J-L$ ). We detected specific binding between the T1 peptide and His-Sytx1A with a $K_{\mathrm{D}}$ of $\sim 3.9 \pm 1.110^{-6} \mathrm{M}\left(k_{\text {on }}, 2.9 \times 10^{4} \mathrm{M}^{-1} \mathrm{~s}^{1} ; k_{\text {off }}, 0.065 \mathrm{~s}^{-1}\right)$. Thus, DCC and Sytx1A interact through specific protein domains.

\section{Netrin-1 regulates the association of DCC and Sytx1}

To determine whether Netrin-1 regulates the association of DCC and Sytx1, we incubated hippocampal cultures with Netrin-1 or control-conditioned medium, and then measured overlapping signals in growth cones by immunofluorescence. Interestingly, Sytx 1 and DCC were localized in distinct vesicle-like organelles in cell bodies and along the shafts of developing axons (Fig. 4A). Weak DCC/Sytx1 colocalization was detected along the leading edges of axonal growth cones (Fig. $4 \mathrm{~B}$ ). These data indicate that the DCC receptor and Sytx1 are transported in different vesicles along axons but may interact in distal growth cones of developing 
A
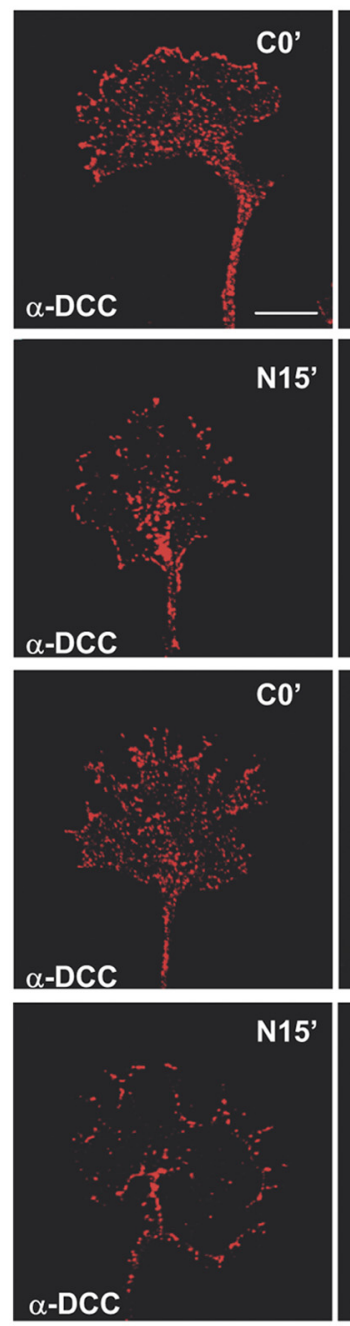
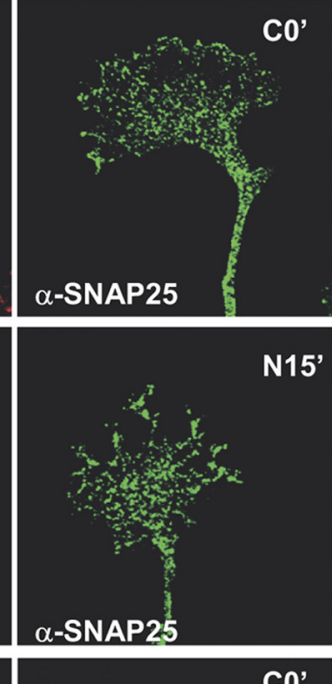

$\alpha$-VAMP2

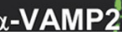

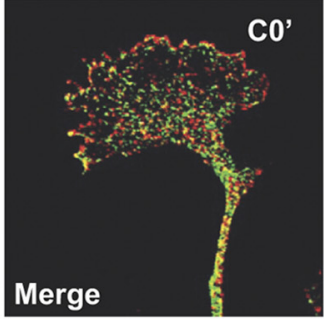

N15,

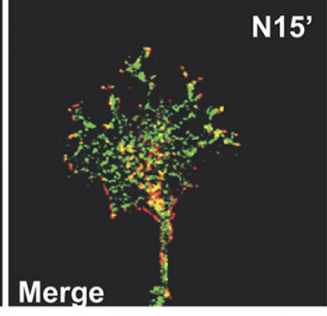

Merge

$\mathrm{CO}^{\prime}$
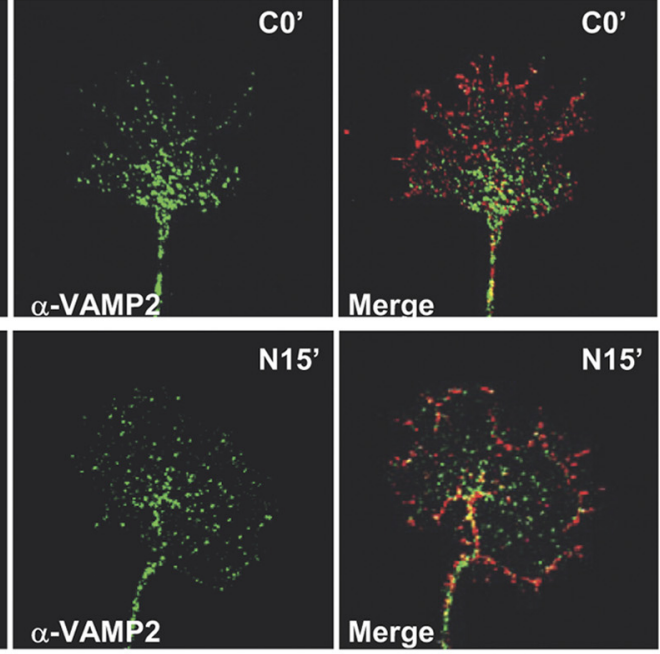
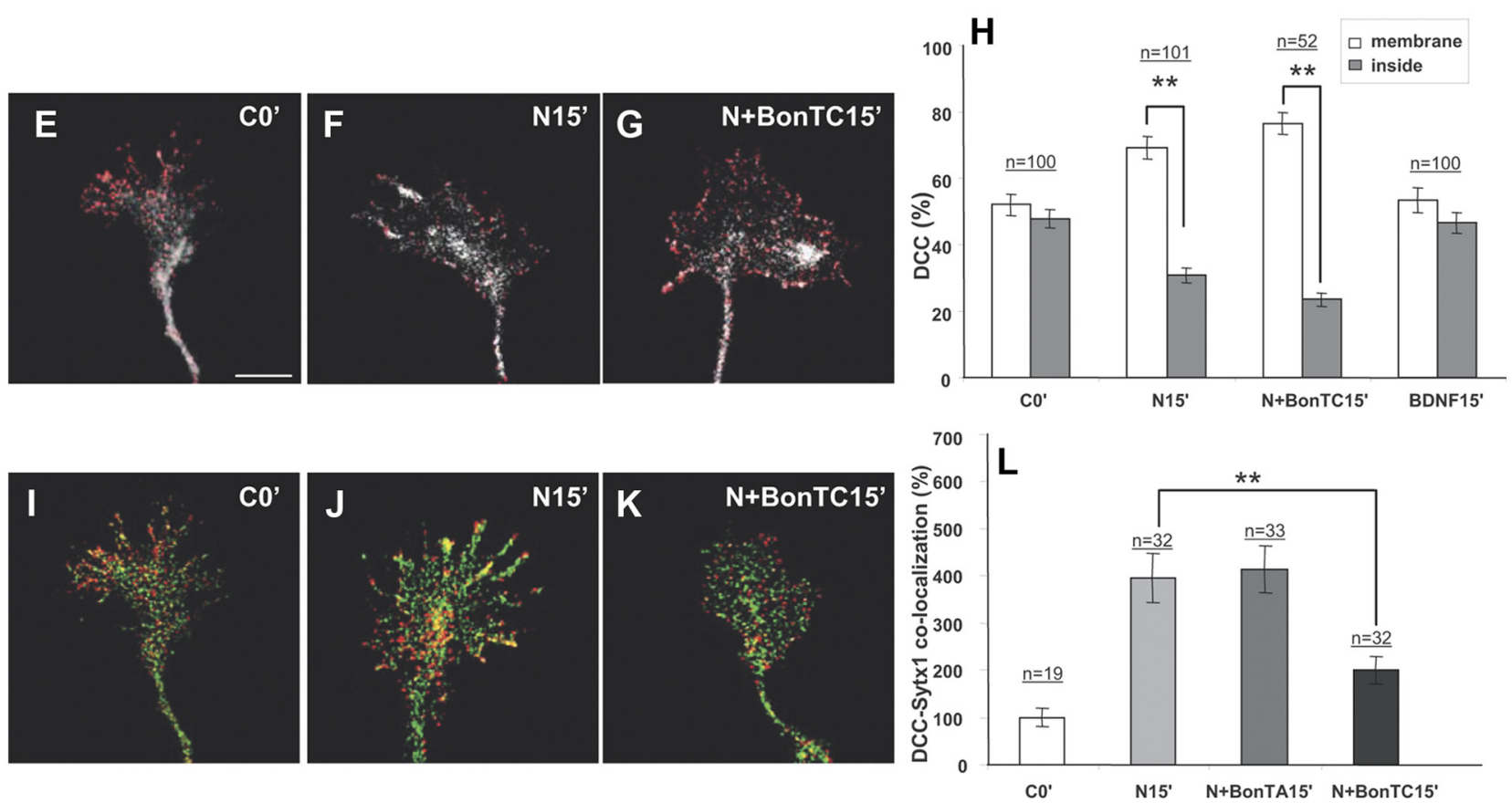

Figure 5. Netrin-1 does not trigger coassociation of DCC with the SNARES SNAP25 and VAMP2. A, Confocal images of hippocampal growth cones treated with Netrin-1-conditioned media for 0 and $15 \mathrm{~min}$, immunolabeled for DCC and the SNAREs SNAP25 and VAMP2. Note low colocalization signals of DCC and SNAP25 or VAMP2, both in control conditions and after (Figure legend continues.) 
neurons. Growth cones treated with Netrin-1 for 15-30 min exhibited a strong increase in DCC/Sytx1 in signal overlapping compared with neurons incubated with control medium (Fig. $4 B, C)$. In contrast, the neurotrophin BDNF, which does not bind DCC, did not increase DCC/Sytx 1 colocalization, indicating that the increase in DCC/Sytx1 colocalization was specifically triggered by Netrin-1 (Fig. $4 E$ ).

To substantiate these findings, lysates of neuronal cultures incubated with Netrin-1 were immunoprecipitated with DCC or Sytx 1 antibodies. Immunoprecipitation with the former revealed higher levels of Sytx1 in cultures incubated with Netrin-1 for 15 and $30 \mathrm{~min}$ than in control cultures. The reverse immunoprecipitations with anti-Sytx1 antibodies also showed higher DCC protein levels after incubation with Netrin-1 (Fig. $4 D, G$ ). Incubation of hippocampal cultures with control media or BDNF did not increase the DCC/Sytx1 coassociation (Fig. 4E,F).

To determine whether the DCC/Sytx1 interaction was dependent on Netrin-1 in vivo, we analyzed lysates from wild-type and netrin-1-deficient mice (Serafini et al., 1996). The amount of DCC protein that was immunoprecipitated with anti-Sytx1 antibodies was severely diminished in netrin-1-deficient forebrains in comparison with wild-type brains. A similar reduction was observed when DCC immunoprecipitates were analyzed for Sytx1 by immunoblotting (Fig. 4G,H).

Interestingly, and in agreement with the above data (Fig. 2), cultured neurons treated with Netrin-1 did not trigger increases in the coassociation of DCC with the SNAREs SNAP25 and VAMP2, as detected by both confocal microscopy and coimmunoprecipitation (Fig. 5A-D). Thus, DCC did not appear to bind VAMP2 and SNAP25 in any of the tested conditions, including incubation with the DCC ligand Netrin-1. We therefore conclude that Netrin-1 specifically promotes the interaction of DCC and Sytx1 in growth cones of cultured neurons.

It has been shown that Netrin-1 triggers membrane exposure of DCC-containing vesicles in growth cones (Bouchard et al., 2004; Moore et al., 2008). First, we corroborated that incubation with Netrin-1, but not with control media or BDNF (which acts via TrkB receptors), caused the mobilization of DCC from the inside of growth cones to the cell membrane (Fig. $5 E-H$ ) (Bouchard et al., 2004). Moreover, we found that treatment with

$\leftarrow$

(Figure legend continued.) incubation with Netrin-1. B, C, Quantification of DCC/SNAP25 and DCC/NAMP2 colocalization signals in hippocampal growth cones (expressed as percentage of DCC/SNARE colocalization over total SNARE signals) in cultures treated with Netrin-1conditioned (black bars) or control-conditioned (white bars) media. Note decreased DCC/SNARE colocalization signals after Netrin-1 treatment. $\boldsymbol{D}$, Western blots from hippocampal cultures treated with Netrin-1- (N) or control- (C) conditioned media for 0-30 min, and immunoprecipitated with anti-DCC or anti-Sytx1A antibodies. Immunoblots reveal no coimmunoprecipitation of DCC with the SNARES SNAP25 and VAMP2 after incubation with DCC. Note coimmunoprecipitation of Sytx1A with SNAP25 and VAMP2. E-G, Confocal images of hippocampal growth cones treated with Netrin-1-conditioned media for 0 and 15 min, and in the presence of BoNT/C1. Cultures were immunolabeled for DCC and stained with phalloidin. Note that the mobilization of DCC to the axonal membrane after incubation with Netrin-1 $(\boldsymbol{F})$ is not altered by BoNT/C1 incubation (G). $\boldsymbol{H}$, Quantification of DCC signals in the periphery of and inside growth cones treated with Netrin-1-conditioned media for 0, 15, and 30 min, showing mobilization of DCC to the axonal membrane after incubation with Netrin-1. Whereas no DCC mobilization is detected after incubation with BDNF, treatment with BoNT/C1 does not alter DCC mobilization. $\boldsymbol{I}-\boldsymbol{L}$, Confocal images of control growth cones $(\boldsymbol{I})$ and cones incubated with Netrin-1-conditioned media for 15 min (J), and with Netrin-1/BoNT/C1 (K). Cultures were immunolabeled for DCC (red) and Sytx1 (green). Note that the increase in DCC/Sytx1 colocalization in $J$ is blocked after incubation with BoNT/C1 (K). L, Histograms illustrating DCC/Sytx1 colocalization in several experimental conditions. Significant differences are labeled by asterisks ( ${ }^{*} p \leq$ $\left.0.05,{ }^{* *} p \leq 0.001\right)$. Scale bar: $A, 3 \mu \mathrm{m}$. Error bars indicate SEM.
BoNT/C1 did not alter the DCC mobilization induced by Netrin-1. Incubation of hippocampal cultures with BoNT/C1 gave a yield of Sytx1 degradation (data not shown) similar to that in Figure $6 E$. In contrast, and interestingly, incubation of hippocampal growth cones with BoNT/C1 prevented the increase in DCC/Sytx1 colocalization induced by Netrin-1 (Fig. 5J-L). This observation indicates that the release of DCC to the membrane is independent of Sytx1 (Fig. $5 E-H$ ). This is consistent with the observation that DCC and Sytx1 do not colocalize in the same vesicles along axonal shafts and in growth cones (see above). Together, these findings indicate that, although the DCC/Sytx1 interaction occurs in the absence of Netrin-1, this guidance cue triggers a specific increase in the association of these proteins.

\section{BoNT/C1 blocks Netrin-1-induced chemoattraction of hippocampal axons}

To study whether Sytx1 is required for Netrin-1-dependent chemoattraction, we first used botulinum toxins, metalloproteases that inhibit the exocytosis of synaptic vesicles and neurotransmitter release by cleaving specific SNARE proteins. While BoNT/A exclusively cleaves SNAP25, BoNT/C1 cleaves Sytx1 and SNAP25 (Blasi et al., 1993a,b; Schiavo et al., 2000). We used hippocampal explants cultured in collagen gels with Netrin-1-expressing cells to examine whether t-SNARE protein cleavage by botulinum toxins interferes with Netrin-1-induced axonal guidance. Cleavage of target t-SNARE proteins in our culture model was confirmed by WB (Fig. $6 E$ ). The amount of cleaved SNARE proteins seen in our culture conditions was similar to that described in previous studies, including mature neurons, and the relatively small amount of Sytx1 cleaved by BoNT/C1 is believed to be due to the fact that Sytx1 protein forming part of SNARE complexes is protected against cleavage (Blasi et al., 1993a,b; Schiavo et al., 2000). We next showed that botulinum toxins did not affect the secretion of Netrin-1 in HEK293 cells stably transfected with a Netrin-1 expression vector (Fig. 6F). Thus, hippocampal explants (Barallobre et al., 2000) were cocultured with aggregates of control or Netrin-1-expressing cells, in the presence of botulinum toxins.

While explants cultured with control cells showed radial axonal growth, those cultured with Netrin-1-expressing cells exhibited axonal attraction (Fig. 6A, $B, G$ ). Chemoattraction was maintained when hippocampal explants were cocultured with Netrin-1 in the presence of BoNT/A (Fig. 6C,G). In contrast, Netrin-1-dependent chemoattraction was impaired in explants incubated with BoNT/C1, which resulted in radial axonal growth (Fig. 6D, G). Quantification of axonal growth by comparing the density of outgrowing axons in the proximal and distal quadrants supported these observations (Fig. 6G).

We also treated hippocampal explants with TeNT, which specifically cleaves the v-SNARE VAMP2 [Fig. 7E (Blasi et al., 1993b)]. Incubation of explants with this toxin did not alter Netrin-1-induced chemoattraction of hippocampal axons (Fig. 7 ), in agreement with the nondetectable coassociation of DCC with VAMP2 (Figs. 2, 5). Together with the above results, the data suggest that, while the SNAREs SNAP25 and VAMP-2 are dispensable for Netrin-1-signaling, Sytx1 is specifically required for Netrin-1-dependent axonal guidance.

\section{Sytx1A is required for commissural axon pathfinding in the chicken spinal cord in vivo}

The requirement for functional Sytx1 in Netrin-1-mediated axonal guidance in vivo was analyzed in the chicken spinal cord, where commissural dorsal axons extend toward the floor 

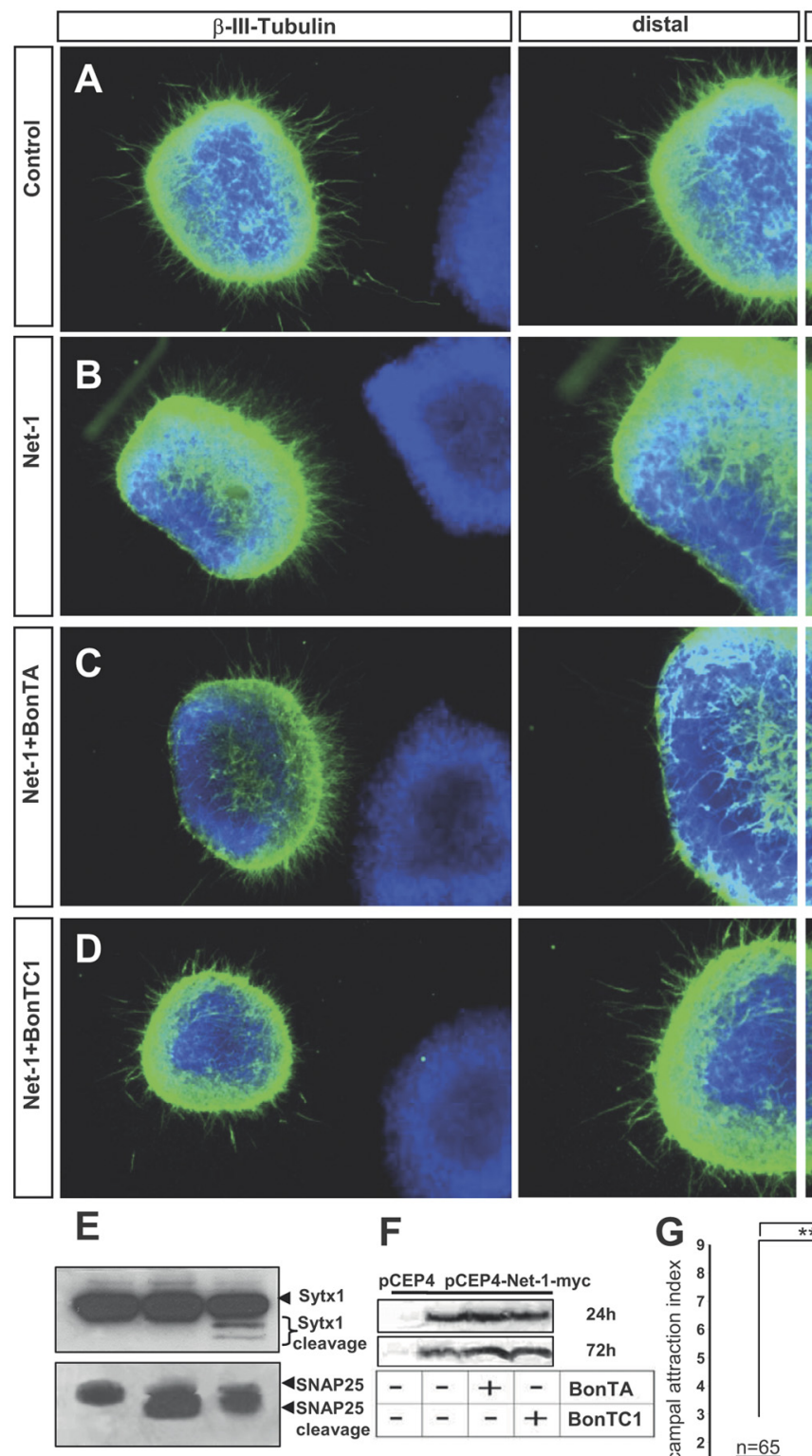

Ctrl BonTA BonTC1

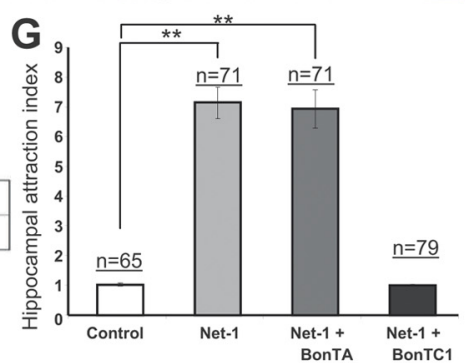

Figure 6. BoNT/C1 blocks Netrin-1-mediated chemoattraction of hippocampal axons. $\boldsymbol{A}-\boldsymbol{D}$, Examples of hippocampal explants cocultured in collagen gels with control or Netrin-1-expressing cell aggregates. Explants cocultured with control cells show a radial growth $(\boldsymbol{A})$. Explants cocultured with Netrin-1-secreting cells exhibit chemoattraction toward the Netrin-1 source $(\boldsymbol{B})$. Explants cocultured with Netrin-1-expressing cells and BoNT/A also display chemoattraction ( $\boldsymbol{C}$. Netrin-1-dependent chemoattraction is blocked in hippocampal explants cultured with BoNT/C1 (D). Low-power images are shown in the first row of panels. High-power magnifications of the proximal and distal quadrants are illustrated in the second and third rows. $\boldsymbol{E}$, Western blots of protein samples from lysates of hippocampal explants cultured with BoNT/A, BoNT/C1, or left untreated (Ctrl), probed with $\alpha$-Sytx1 and $\alpha$-SNAP25 antibodies. Incubation with BoNT/A results in cleavage of SNAP25 and treatment with BoNT/C1 cleaves both SNAP25 and Sytx1. F, Western blots of conditioned media from control HEK293 cells or cells stably transfected with pCEP4-Netrin-1-myc cultured for $24-48 \mathrm{~h}$ in the presence or absence of BoNT/A or BoNT/C1, probed with an $\alpha$-Myc antibody to detect Netrin- 1 in the culture media. The Western blot did not show any differences in the secretion of Netrin-1 in the presence of botulinum toxins. $\mathbf{G}$, Histograms illustrating proximal/distal ratios (attraction index) in hippocampal explants in different culture conditions. Significant differences are labeled by asterisks ( $\left.{ }^{* *} p \leq 0.001\right)$. Scale bar: $\boldsymbol{A}, 300 \mu \mathrm{m}$. Error bars indicate SEM.

plate in a Netrin-1- and DCC-dependent manner (Serafini et al., 1996; Fazeli et al., 1997). To assess the relevance of Sytx1A, we first expressed dominant-negative constructs (pIRES-Sytx $1 \mathrm{~A}_{\mathrm{H} 3 \mathrm{TM}} \mathrm{EGFP}$ or the fusion Sytx1 $\mathrm{A}_{\mathrm{HзтM}} \mathrm{EGFP}$ ) in commissural neurons at the time when they start to extend axons. After $2 \mathrm{~d}$, the trajectory of commissural axons was visualized in transverse slices or in whole-mount
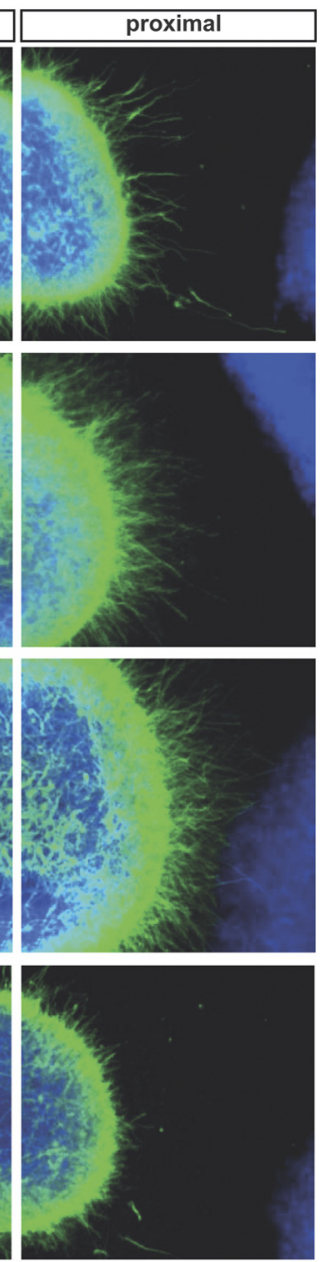

preparations of the spinal cord, the so-called "open-book preparations" (Fig. 8J), by the injection of Fast DiI (Perrin and Stoeckli, 2000). In untreated control spinal cords, virtually all commissural axons had crossed the floor plate. After entering the floor plate and crossing the midline, these axons turned into the longitudinal axis of the spinal cord and extended rostrally, while maintaining contact with the floor plate border (Fig. $8 A, B)$. However, and in contrast to commissural axons from embryos transfected with the control pIRES-EGFP construct, those transfected with the dominantnegative pIRES-Sytx1 $\mathrm{A}_{\mathrm{H} 3 \mathrm{TM}}$ EGFP failed to cross the floor plate and stalled before or at the floor plate border (Fig. 8C-F). In fact, in pIRES-Sytx1 $\mathrm{A}_{\mathrm{H} 3 \mathrm{TM}}$ EGFP-electroporated spinal cords, many fibers failed to reach the floor plate and extended along the lateral edge of the spinal cord (Fig. $8 E$, arrowhead). The same results were obtained with the Sytx $1 \mathrm{~A}_{\mathrm{H} 3 \mathrm{TM}}$ EGFP fusion protein both qualitatively (data not shown) and quantitatively (Fig. $8 \mathrm{~K}$ ). While embryos expressing the Sytx $1 \mathrm{~A}_{\mathrm{TM}} \mathrm{EGFP}$ fusion protein did not show a significant increase in the failure of commissural axons to reach and cross the floor plate, expression of Sytx $1 \mathrm{~A}_{\mathrm{H} 3}$ EGFP resulted in a mild phenotype (Fig. $8 \mathrm{~K}$ ).

As an independent approach to interfere with Sytx1A function in commissural axon pathfinding, we used in ovo RNAi (Pekarik et al., 2003). Downregulation of Sytx1A in commissural neurons by electroporation of dsRNA derived from Sytx1A gave similar results to the perturbation of Sytx1A function by dominant-negative Sytx1A variants. Thus, downregulation of Sytx1A by in ovo RNAi resulted in the failure of commissural axons to reach and cross the floor plate (Fig. $8 G, H, K)$. As a control, we used dsRNA derived from $\mathrm{NgCAM}$, a cell adhesion molecule of the Ig superfamily that is expressed in dorsal commissural neurons (Pekarik et al., 2003). Perturbation of NgCAM function interfered with the fasciculation of commissural axons but not with their midline crossing (Fig. $8 I, K$ ). In summary, we found strong interference with commissural axon pathfinding toward and across the floor plate after in vivo perturbation of Sytx1A function either by expressing dominant-negative variants of Sytx1A or by its knockdown using in ovo RNAi. Moreover, the phenotypes observed here are reminiscent of those described in loss-of-function models of the Netrin-1 and DCC genes (Serafini et al., 1996; Fazeli et al., 1997).

\section{Netrin-1 triggers exocytosis in growth cones}

We next studied whether Netrin-1 regulates exocytosis in growth cones through a DCC/Sytx1 interaction. For this purpose, hip- 

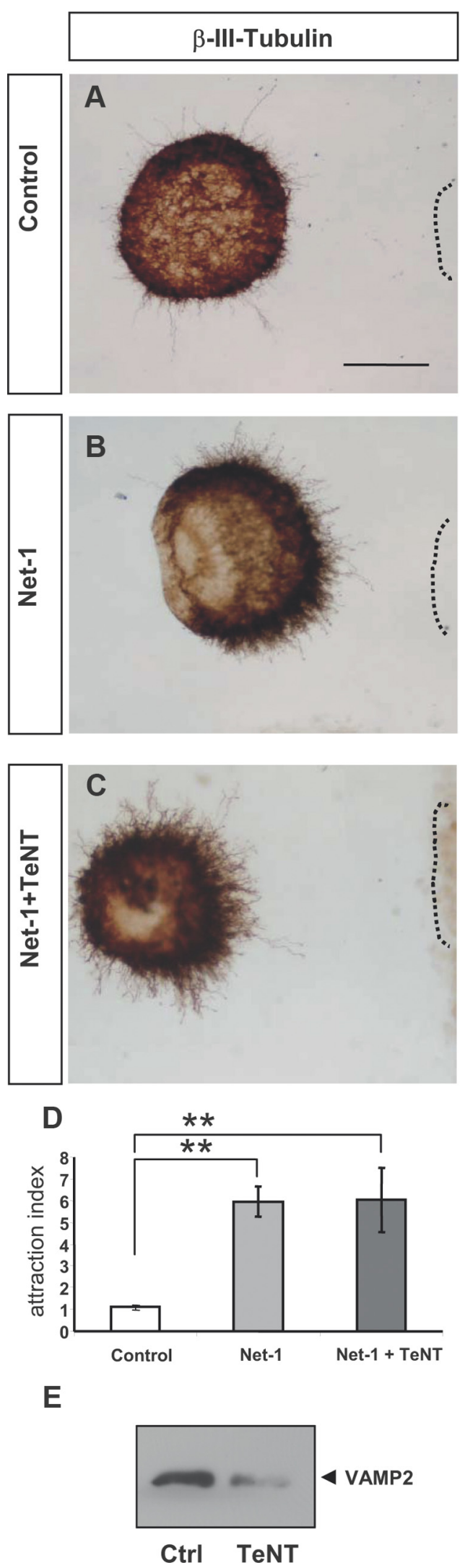

Figure 7. TeNT incubation does not alter Netrin-1-induced chemoattraction. $A-C$, Examples of hippocampal explants cocultured with control or Netrin-1-expressing cell aggregates in the presence of TeNT. Explants were immunolabeled for $\beta$ III-tubulin. Explants cocultured with Netrin-1-secreting cells show strong chemoattraction $(\boldsymbol{B})$. Explants cocultured with Netrin-1-secreting cells and TeNT also show strong chemoattraction (C). D, Histogram illustrating proximal/distal ratios (attraction index) in a range of conditions. Hippocampal explants pocampal cultures were prelabeled with BODIPY ceramide (a membrane, lipid marker that preloads vesicular compartments) (Pfenninger et al., 2003; Pfenninger, 2009), and then incubated with Netrin-1 at a range of times. The decrease in fluorescence in BODIPY ceramide-preloaded growth cones reflects exocytosis (Pfenninger et al., 2003). While incubation with control media did not result in a decrease of BODIPY ceramide signals in growth cones, treatment with Netrin-1 led to a substantial reduction of intracellular BODIPY ceramide fluorescence, thereby indicating release of prelabeled vesicles (Fig. 9A, $B, D$ ). Moreover, incubation with BoNT/C1, 30 min before Netrin-1 stimulation, abolished the decrease in fluorescence, thus suggesting blockade of vesicle release (Fig. 9C,E). In contrast, incubation of growth cones with BoNT/A or TeNT did not decrease the vesicle release induced by Netrin-1 (Fig. 9E). These observations strongly suggest that Netrin-1 triggers local exocytosis in growth cones and that Sytxl is required for these events.

\section{The v-SNARE TI-VAMP interacts with DCC and is required for commissural axonal guidance in vivo}

In addition to the t-SNARE Sytx1, vesicle exocytosis and membrane fusion require the formation of a protein complex with v-SNAREs. As described above, we tested whether DCC coassociates with the classical neural SNARES that regulate neurotransmitter release. However, coimmunoprecipitation assays revealed that DCC does not interact with SNAP25, VAMP2, or Sytx4 (a SNARE involved in protein traffic) (Figs. 2, 5). This observation is in agreement with the finding that BoNT/A and TeNT, toxins that block SNAP25 and VAMP2, respectively, did not affect Netrin-1/DCC-dependent guidance (Figs. 6, 7).

The v-SNARE TI-VAMP is selectively enriched in growth cones and has been proposed to mediate exocytotic events involved in neurite growth (Alberts et al., 2006). We thus studied whether TI-VAMP forms part of the DCC/Sytx1 complex. Cultured hippocampal neurons exhibited colocalization of DCC and TI-VAMP, which was conspicuous at the edges of the growth cones (Fig. 9F). Expression of DCC and TI-VAMP constructs in EBNA293 cells followed by coimmunoprecipitation and WB showed that these two proteins interact in vitro after heterologous expression, both in the presence or absence of Sytx1 (Fig. 9G). In contrast, TI-VAMP did not coassociate with Frizzled 2, another membrane receptor (Fig. 9G). Furthermore, DCC and TI-VAMP coimmunoprecipitation experiments from E15 and adult brain lysates demonstrated that DCC interacts with TI-VAMP in vivo (Fig. $9 H$ ). In agreement with the low expression of TI-VAMP in adult tissue, DCC/TI-VAMP interaction was diminished in adult brain. These two proteins also interacted with Sytx1, whereas control immunoprecipitations with an irrelevant antibody in brain lysates were negative (Fig. $9 H$ ). These findings show that, in contrast to VAMP2, the vesicular membrane protein TI-VAMP belongs to the DCC/Sytxl complex, suggesting that this v-SNARE mediates Netrin-1-dependent guidance.

To examine this possibility, we studied the impact of TI-VAMP downregulation in the development of Netrin-1dependent commissural pathway in the chicken spinal cord. TI-

$\leftarrow$

cultured with Netrin-1-expressing cells in the presence or absence of TeNT show chemoattrac tive patterns of axonal growth. $\boldsymbol{E}$, Western blots of protein samples from lysates of hippocampal explants cultured with TeNT, or left untreated (Ctrl), probed with $\alpha$-VAMP2 antibody. Incubation with TeNT cleaves VAMP2. Significant differences are labeled by asterisks ( $\left.{ }^{* *} p \leq 0.001\right)$. Scale bar: $\boldsymbol{A}, \boldsymbol{D}, 150 \mu \mathrm{m}$. Error bars indicate SEM. 

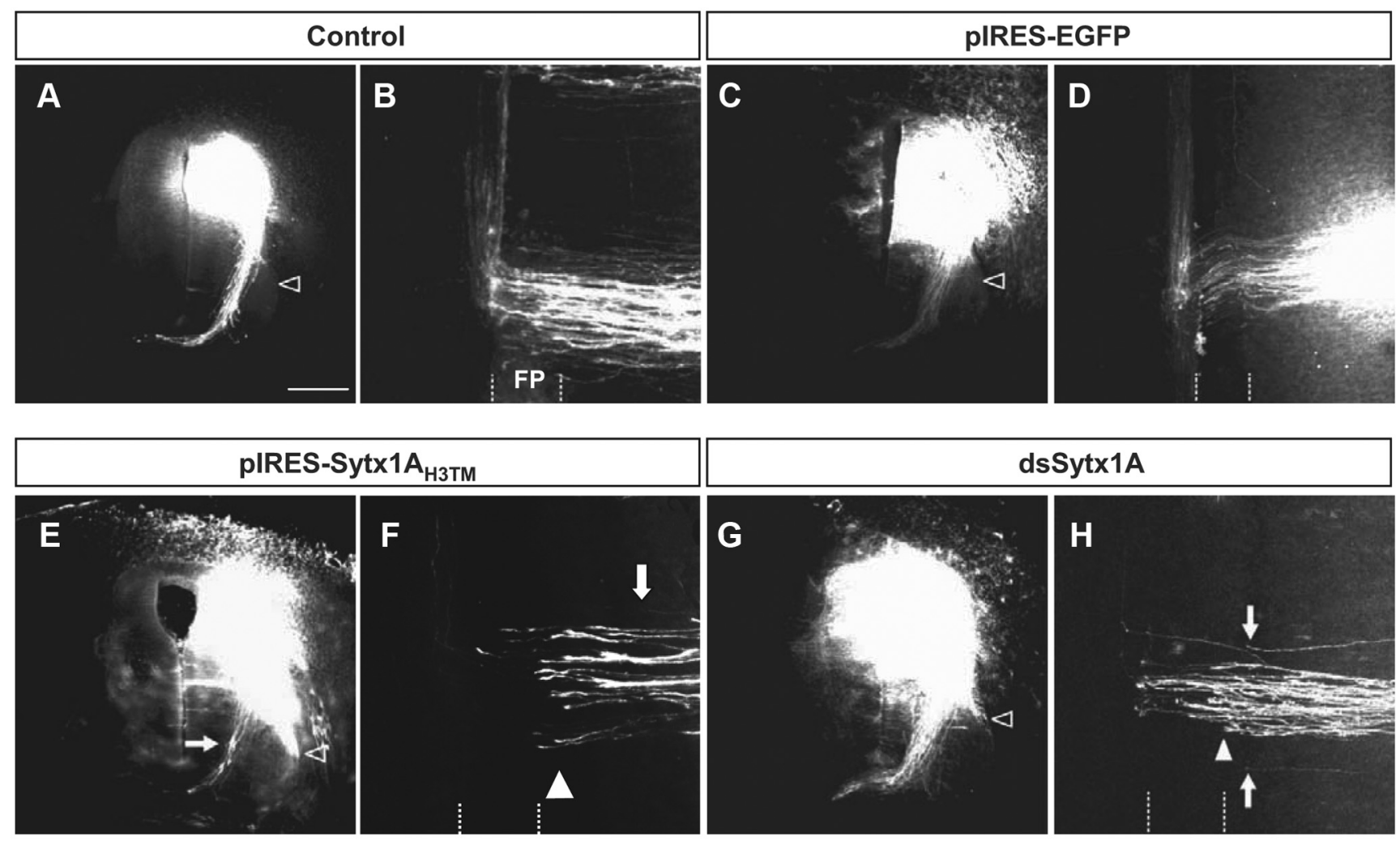

dsSytx1A
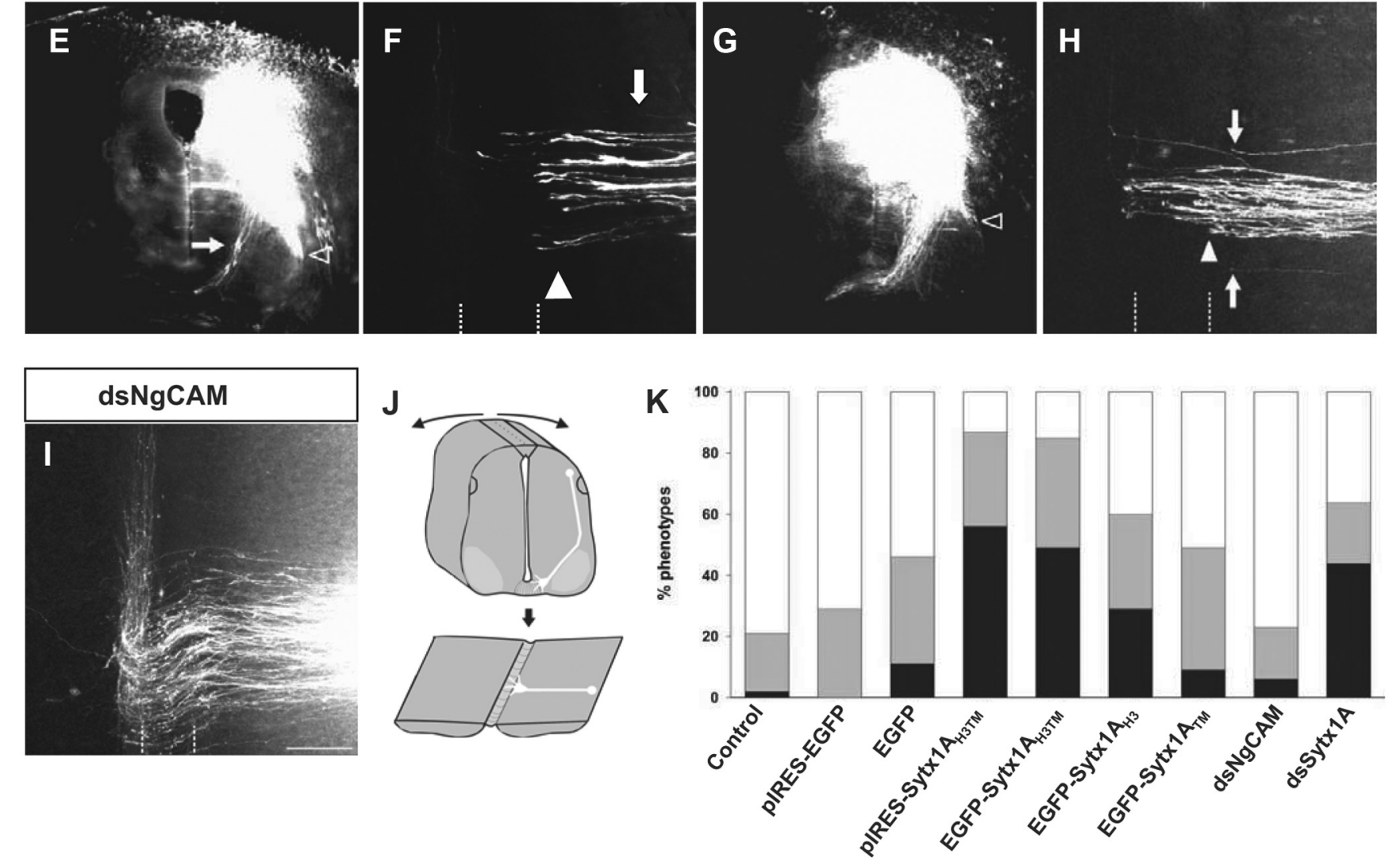

Figure 8. Sytx1A function is required for the navigation of dorsal commissural axons toward and across the floor plate in vivo. $A, C, E$, and $G$ are transverse sections. $B, D, F, H$, and $I$ show open-book preparations of the spinal cord that were obtained by cutting the roof plate and flipping the cord open as indicated by the arrows (D). Rostral is at the top of the panels. $A, B$, Dorsolateral commissural neurons extend their axons toward the floor plate in a Netrin-1-dependent manner. By stage 25, axons have crossed the floor plate and turned into the longitudinal axis of the spinal cord, and are still in contact with the floor plate. $\boldsymbol{C}, \boldsymbol{D}$, The injection of the pIRES-EGFP construct did not interfere with commissural axon pathfinding. $\boldsymbol{E}-\boldsymbol{H}$, Perturbation of Sytx1A function either by expression of the dominant-negative variant $\operatorname{Sytx} A_{\text {нзтм }}(\boldsymbol{E}, \boldsymbol{F})$ or by its downregulation using in ovo RNAi $(\boldsymbol{G}, \boldsymbol{H})$ interfered with commissural axon navigation to and across the floor plate. A large number of axonsfailed to reach the floor plate $(\boldsymbol{E}, \boldsymbol{F}, \boldsymbol{H}$, arrows) or stopped at the ipsilateral floor plate border $(\boldsymbol{F}, \boldsymbol{H}$, arrowhead). As would be expected in the absence of Netrin-1-mediated axon guidance, axons were found to extend ventrally along the pial surface of the neural tube rather than along the correct pathway $(\boldsymbol{E}, \boldsymbol{G}$, open arrowhead). Axons were never found to extend along the spinal cord margin in control embryos ( $A, C$, , open arrowhead). $I, A s$ a control for the specificity of RNAi, we used dsRNA from NgCAM. NgCAM is a cell adhesion molecule of the lg superfamily expressed by commissural axons. NgCAM was shown to be required for fasciculation of commissural axons but not for their midline crossing. $\boldsymbol{K}$, Quantification of the axon guidance phenotype in open-book preparations of embryonic chicken spinal cords. Phenotypes were assessed in open-book preparations (black bars, strong phenotype; gray bars, weak phenotype; white bars, normal phenotype). We analyzed at least 10 embryos per condition (except for pIRES) with $6-10$ dye injection sites per spinal cord. A failure to reach and cross the floor plate was found at $85 \%$ of the injection sites of embryos electroporated with the Sytx1H3TMEGFP fusion protein (91 injection sites in 10 embryos with $48 \%$ strong and $36 \%$ weak phenotypes). Similarly, the expression of pIRES-Sytx1AH3TMEGFP resulted in a phenotype at $87 \%$ of the injection sites ( 94 injection sites in 12 embryos with $56 \%$ strong and $31 \%$ weak phenotypes). In contrast, using the same criteria, embryos expressing the EGFP under the control of the $\beta$-actin promoter ( 74 injection sites in 11 embryos with $11 \%$ strong and $35 \%$ weak phenotypes) or from the pIRES construct (51 injection sites in 7 embryos; $71 \%$ of them normal; $29 \%$ with a weak phenotype) did not show a significant increase in the failure of commissural axons to reach and cross the floor plate. The same result was also found for the expression of the Sytx1ATMEGFP fusion protein (85 injection sites in 11 embryos with $9 \%$ strong and $40 \%$ weak phenotypes). We found a weak but significant increase in axon guidance errors in embryos expressing the Sytx1AH3EGFP fusion protein. A phenotype was observed at 60\% of the injection sites ( 80 injection sites in 11 embryos with 29\% strong and 31\% weak phenotypes) in Sytx1AH3EGFPexpressing embryos. Downregulation of Sytx1A by in ovo RNAi resulted in the failure of commissural axons to reach and cross the floor plate at $64 \%$ of the injection sites (96 injection sites in 12 embryos with $44 \%$ strong and $20 \%$ weak phenotypes). Perturbation of $\mathrm{NgCAM}$ function was shown to interfere with fasciculation of commissural axons but not with their midline crossing. Accordingly, we found successful crossing of the floor plate at $77 \%$ of the injection sites ( 87 injection sites in 12 embryos with only $6 \%$ of the injection sites exhibiting a strong phenotype). Scale bars: $A, 150 \mu \mathrm{m} ; I, 50 \mu \mathrm{m}$. 

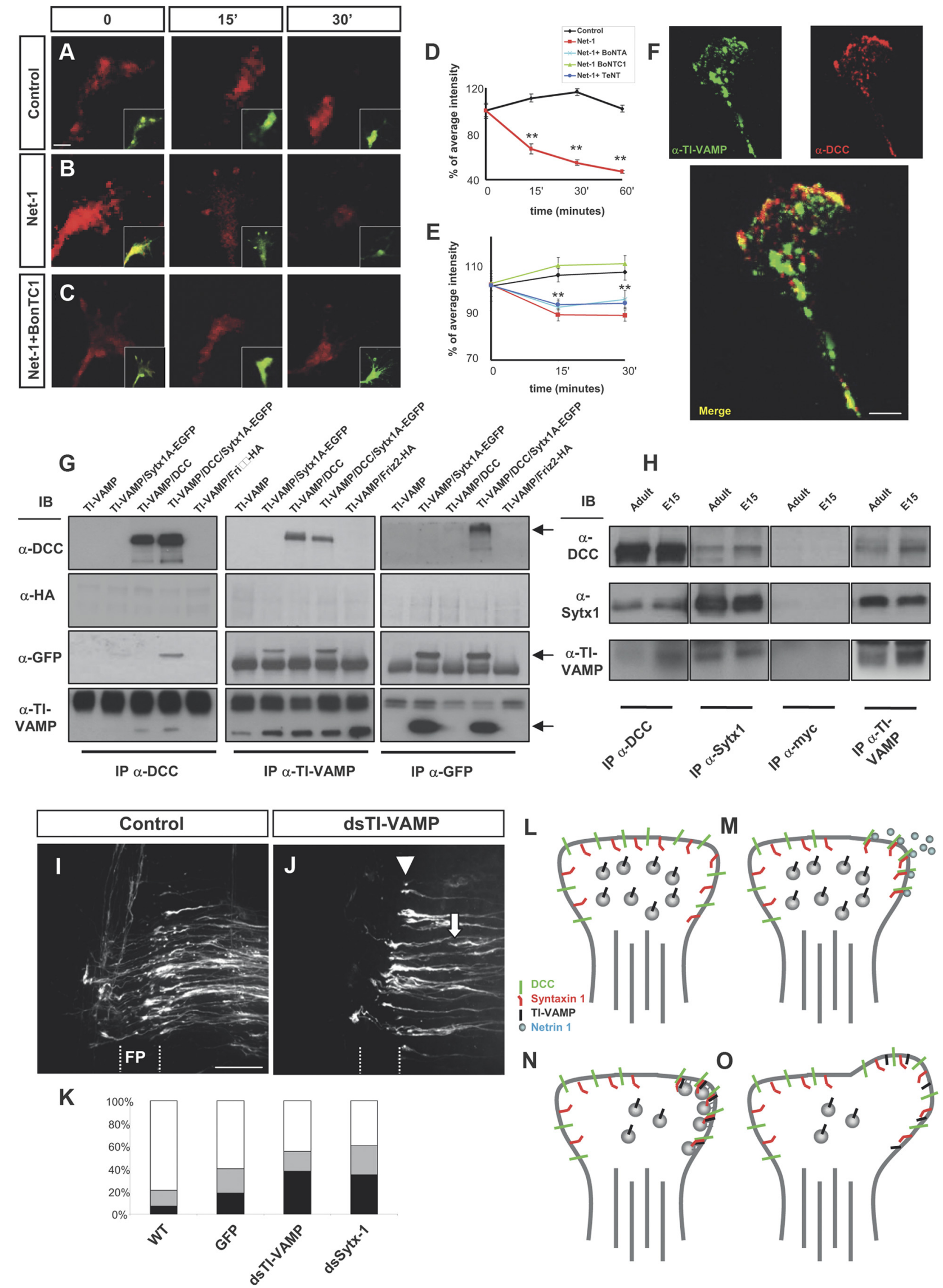

Figure 9. Netrin-1 elicits exocytosis and axonal guidance depending on Sytx1 and TI-VAMP. A-C, Confocal images of live hippocampal growth cones traced with BODIPY (recorded in the red channel). Neurons were labeled with BODIPY ceramide for $30 \mathrm{~min}$ at room temperature and then chased for $2.5-3 \mathrm{~h} \mathrm{at} 37^{\circ} \mathrm{C}$. Growth cones were treated with control (Figure legend continues.) 
VAMP knockdown resulted in many commissural axons stopping either before entering the floor plate or in the midline itself, and thus in a fiber phenotype that was similar to that observed after Sytx1 downregulation (Fig. 9I-K; see also Fig. $8 \mathrm{~K}$ ). Together, these data indicate that a DCC/Sytx1/TI-VAMP protein complex is required to mediate Netrin-1-dependent axonal guidance.

\section{Discussion}

Binding of Netrin-1 to the DCC receptor activates several intracellular kinase cascades including the MAPK pathway and the SFK (Src family kinase)/FAK (focal adhesion kinase) pathways required for growth cone guidance (Ming et al., 1999; Forcet et al., 2002). In addition, rearrangement and stabilization of cytoskeletal proteins is a critical step for Netrin-1-dependent chemotropism in developing axons. For example, Netrin-1 chemoattraction requires activation of the small GTPase Rho, but not of Rac (Li et al., 2002; Causeret et al., 2004), and formation of Ena/VASP (vasodilatorstimulated phosphoprotein) complexes that interact with actin ultimately leading to actin polymerization (Gitai et al., 2003; Lebrand et al., 2004). It has recently been shown that neurotrophins and

$\leftarrow$

(Figure legend continued.) medium, Netrin-1, or Netrin-1 plus BoNT/C1 for $0-30$ min. While fluorescent vesicle clusters persist in controls $(\boldsymbol{A})$, red fluorescence rapidly disappears upon treatment with Netrin- $1(\boldsymbol{B})$, indicating the occurrence of secretion events. Treatment with BoNT/C1 prevents the disappearance of red puncta in Netrin-1-treated neurons $(\boldsymbol{C})$. On the bottom right, merged images showing the BODIPY label recorded separately in the red (high concentration) and green (low concentration) channels. The red spots show Golgi-derived vesicles or vesicle clusters, whereas the green fluorescence outlines the plasmalemma of the growth cone, labeled with small amounts of BODIPY.D, E, Percentage of average intensity of red fluorescent puncta in growth cones for control and Netrin-1-treated hippocampal neurons (normalized to $100 \%$ at the onset of treatment) $(\boldsymbol{D})$. The average intensity of spots recorded in the red channel comes back to control levels after treatment with BoNT/C1 (E). $\boldsymbol{F}$, Confocal images of hippocampal growth cones immunolabeled for DCC and TI-VAMP. A merged image showing colocalization of both proteins is shown at the bottom. G, Coimmunoprecipitation experiments in HEK293 cells transfected with pCMV6TI-VAMP and Sytx1AEGFP, pCMVDCC, or Friz2-HA DNAs. $D C C$ immunoprecipitation results in coassociation with Sytx1A, visualized with anti-GFP antibodies, and with TI-VAMP (anti-TI-VAMP antibodies). The reverse immunoprecipitation with anti-TI-VAMP antibodies confirms DCC and Sytx 1 coimmunoprecipitation. GFP immunoprecipitation of Sytx 1 reveals coassociation with DCC and TI-VAMP proteins. Immunoblots show no coimmunoprecipitation of Friz-2 with DCC and the SNAREs Sytx1 and TI-VAMP. H, Coimmunoprecipitation experiments in E15 and adult brain lysates. Sytx 1 and TI-VAMP immunoprecipitation yields coimmunoprecipitation of DCC in both samples. DCC and TI-VAMP immunoprecipitation yields coassociation of Syt $x 1$. DCC and Syt $x 1$ immunoprecipitation yields coassociation of TI-VAMP. Immunoprecipitations with anti-myc antibody were used as controls. $I, J$, Confocal images showing open-book preparations from the chicken spinal cord. The injection of the control pIRES-EGFP construct did not interfere with commissural axon pathfinding (I). Downregulation of TI-VAMP using in ovo RNAi ( $)$ interfered with commissural axon navigation to and across the floor plate, with many fibers being arrested before (arrows) or within the floor plate (FP) (arrowheads). $\boldsymbol{K}$, Quantification of the axon guidance phenotype in openbook preparations of embryonic chicken spinal cords. RNAi-mediated downregulation of TIVAMP in chicken spinal cords ( $n=139$ injection sites in 17 embryos) yielded similar commissural pathfinding errors to those obtained with silencing of Sytx 1 ( $n=135$ injection sites in 18 embryos). We observed strong phenotypes at $37.9 \%$ of dsTI-VAMP and $34.6 \%$ of dsStx-1, and weak phenotypes at 17.2 and $25.7 \%$ of injection sites, respectively. By contrast, electroporation of a pIRES plasmid expressing EGFP under the $\beta$-actin promoter (297 injection sites in 28 embryos; $60.1 \%$ normal and $21 \%$ weak phenotypes) did not differ significantly from untreated controls (6.9\% strong phenotypes, $13 \%$ weak; $n=489$ injection sites in 47 embryos). $\mathbf{L} \mathbf{- 0}$, Schematic diagram summarizing a model for the regulation of Netrin-1dependent exocytosis in growth cones. In a steady-state situation, the growth cones present a low release of exocytosis vesicles $(\boldsymbol{L})$. Netrin-1 activation of DCC receptors result in liganddependent clustering of DCC/Sytx1 complexes in activated membrane domains $(\boldsymbol{M})$. Here, the formation of a SNARE complex between Sytx 1 and TI-VAMP proteins occurs, thereby promoting exocytosis of vesicles at $D C C$-activated domains $(\boldsymbol{N})$, resulting in membrane expansion $(\mathbf{O})$. Significant differences are labeled by asterisks $\left({ }^{* *} p \leq 0.001\right)$. Scale bars: $A, 2 \mu \mathrm{m} ; \boldsymbol{F}, 3 \mu \mathrm{m} ; \boldsymbol{I}$, $50 \mu \mathrm{m}$. Error bars indicate SEM.
Netrin-1 increase the transport to growth cones, and the local asymmetrical translation of $\beta$-actin mRNA (Campbell and Holt, 2001; Zhang et al., 2001; Leung et al., 2006; Yao et al., 2006). Moreover, Netrin-1 triggers microtubule assembly (Dent et al., 2004), as well as phosphorylation of MAP1B, a microtubule-associated protein that mediates cross talk between microtubules and actin (Del Río et al., 2004). All these findings indicate that growth cone chemotropic responses require translation and stabilization of cytoskeletal components in the direction of axonal growth.

Another essential component of growth cone dynamics and cell migration is membrane turnover and exocytosis (Craig et al., 1995; Deitcher, 2002; Sabo and McAllister, 2003). The present data showing that BoNT/C1, but not BoNT/A or TeNT, abolishes Netrin-1 chemoattraction in hippocampal axons, strongly support that axons require Sytx1, but not SNAP25 or VAMP2, to read the Netrin-1 signal. These observations are supported by the following: (1) biochemical experiments showing that DCC does not interact with VAMP2 or SNAP25, neither before or after incubation with Netrin-1; (2) biochemical and biophysical experiments demonstrating interaction of DCC with Sytx1A by using complementary techniques including coimmunoprecipitation, pull downs, in vitro translation, and plasmon resonance; (3) the regulation of DCC/Sytx1 coassociation and colocalization by Netrin-1 in protein lysates and growth cones, respectively; (4) experiments showing that Sytx1A dominant-negative constructs and knockdown of Sytx1A interfere with Netrin-1-mediated guidance in vivo during the development of commissural axons in the spinal cord; and (5) data showing that Netrin-1 triggers local exocytosis in growth cones in a Sytx1-dependent manner. Moreover, the present study shows that the Netrin-1 receptor DCC interacts with Sytx 1 through specific protein domains. These biochemical and functional data provide evidence that Sytxl is specifically involved in chemotropic Netrin-1 signaling through direct interaction with the DCC receptor.

Some studies report that BoNT/C1 causes growth cone collapse and neuronal death (Deitcher, 2002; Long et al., 2004), while others describe that this toxin does not affect neuronal viability (Hong et al., 2000; Ming et al., 2002). In the experimental conditions used here (brain explants, and the minimal toxin concentration at which they were found to cleave SNARE proteins), we found no evidence of neurodegeneration in explants or in dissociated cultures. Indeed, neurons grew and extended axons in a similar manner as in control cultures, thereby demonstrating not only that neurons were viable but also that the machinery for basal neurite and axonal extension was preserved. Moreover, in the conditions used here, incubation with BoNT/C1 did not cause growth cone collapse (Fig. 5G).

The phenotypes observed after perturbing Sytx 1 function are consistent with pathfinding deficits. Thus, Netrin-1-dependent chemoattraction was severely impaired in tissue explants and perturbation of Sytx1 in vivo caused phenotypes (arrest of most fibers before crossing the floor plate) similar to those observed in Netrin-1/DCC mutants and knockdowns (Serafini et al., 1996; Fazeli et al., 1997). These observations have led us to propose that Sytx1 is specifically involved in Netrin-1 signaling to regulate exocytosis and guidance through direct interaction with the DCC receptor. This view is consistent with reports indicating that axonal growth and pathfinding are apparently normal in SNAP25- and VAMP2-deficient mice (Osen-Sand et al., 1993; Washbourne et al., 2002) and in Drosophila embryos lacking the SNAP25 ortholog (Rao et al., 2001; Vilinsky et al., 2002). Conversely, several developmental defects are found in Drosophila mutants for the ortholog of Sytx1A, including compaction of the ventral nerve cord, defects in axonal morphology and fasciculation, and a reduction in the number of 
synapses (Schulze et al., 1995; Schulze and Bellen, 1996). However, it was recently reported that Sytx1A-deficient mice do not display neuroanatomical or basal neurotransmission differences compared with control littermates, although the former had impaired long-term and conditioned fear memory (Fujiwara et al., 2006). This observation suggests functional redundancy of Sytx1A with Sytx1B or other Syntaxin family members, as it has been reported for SNAP25 and VAMP2 knock-out mice (Bhattacharya et al., 2002; Vilinsky et al., 2002).

SNARE proteins are essential in $\mathrm{Ca}^{2+}$-dependent exocytosis and neurotransmitter release in adult nervous tissue (Rizo and Südhof, 2002; Jahn and Scheller, 2006; Südhof and Rothman, 2009). These proteins have also been implicated in most secretion events of non-neuronal cells, including developmental processes (Deitcher, 2002). Here, we show that the t-SNARE Sytx1 is specifically required for Netrin-1-induced guidance of axons and migrating neurons through a mechanism that involves Netrin-1dependent binding of Sytx 1 to the Netrin-1 receptor DCC and the v-SNARE TI-VAMP (Fig. 9L-O). Netrin-1 attraction requires high $\mathrm{Ca}^{2+}$ currents, which are caused by activation of TRPC, L-type, and intracellular ryanodine receptor channels (Hong et al., 1999, 2000; Ming et al., 2002; Wang and Poo, 2005). Such $\mathrm{Ca}^{2+}$-activated currents may contribute to SNARE-mediated exocytosis and the extension of growth cones in response to chemotropic substances. These findings suggest a model in which Netrin-1/DCC signaling regulates exocytosis in growth cones by activating two convergent mechanisms, namely, the recruitment of Sytx1 in "activated" membrane domains of the growth cone through ligand-dependent DCC activation, and local Netrin-1/DCC-dependent $\mathrm{Ca}^{2+}$ increases that trigger exocytosis of plasmalemma precursor vesicles and axonal growth in Sytx1-enriched, "activated" membrane domains (Fig. 9L-O).

As the SNAREs VAMP2 and SNAP25 are not recruited to the DCC/Sytx 1 complex (and loss of SNAP25 and VAMP2 do not affect Netrin-1 guidance) (Figs. 5, 6), Netrin-1-mediated axonal growth is likely to involve mechanisms other than the formation of classical SNARE complex (Sytx1/SNAP25/VAMP2) described in adult synapses, responsible for neurotransmitter release (Verhage et al., 2000; Castillo et al., 2002; de Wit et al., 2009; Maximov et al., 2009; Ramakrishnan et al., 2009). In this context, it is interesting to note that high $\mathrm{Ca}^{2+}$ concentrations trigger SNAREmediated exocytosis in a SNAP25-independent manner (Molgó et al., 1989). Examples of atypical SNARE complexes include complexes containing TI-VAMP during neuritogenesis (Danglot et al., 2010), classical SNARE-independent exocytosis in cochlear hair cells (Nouvian et al., 2011), and atypical SNARE complexdependent exocytosis in dendrites (Kennedy and Ehlers, 2011). Here, we show that the TeNT-insensitive protein TI-VAMP, which has previously been shown to be involved in the formation of neurites (Alberts et al., 2006), associates with the DCC/Sytx1 complex and that loss-of-function of this protein results in abnormal Netrin-1-dependent guidance in vivo. In addition to its interaction with SNAP25/VAMP2 SNARE complexes, Sytx1 also interacts with a wide range of membrane proteins including regulators of exocytosis, such as Complexins, Synaptotagmins, Munc-18, RIM, and ionic channels (Verhage et al., 2000; Castillo et al., 2002; de Wit et al., 2009; Maximov et al., 2009; Ramakrishnan et al., 2009). Thus, the molecular and biophysical mechanisms by which DCC may regulate Sytx1/TI-VAMP complexes and exocytosis remains to be elucidated. It is equally possible that the principal role of DCC receptors is the recruitment of Sytx1/ TI-VAMP complexes (Sieber et al., 2007) to the Netrin-1activated membrane domains, thereby facilitating exocytosis in these regions of the growth cone.
"Attractive" $\mathrm{Ca}^{2+}$ signals applied through photoactive compounds elicit asymmetric exocytosis and growth cone turning in a mechanism that depends on v-SNARE VAMP2 (Tojima et al., 2007). Conversely, clathrin-mediated endocytosis is required for Semaphorin 3A-induced chemorepulsion in growth cones (Tojima et al., 2010). Here, we show that Netrin-1 triggers exocytosis in a Sytx1-dependent manner, whereas the Netrin-1-regulated exposure of DCC requires protein kinase A activation and Rho inhibition (Bouchard et al., 2004; Moore et al., 2008) but not Sytx1 (present results). Together with these data, our findings suggest that chemotropic receptor signaling triggers either exocytosis or endocytosis, depending on whether guidance receptor activation triggers attraction or repulsion, respectively. Moreover, the present experiments point to a scenario in which distinct extracellular cues activate parallel signaling pathways, requiring either VAMP2 or TI-VAMP, to regulate local exocytosis in growth cones by parallel mechanisms. The activation of these pathways may depend on the capacity and properties of distinct axonal guidance receptors to coassociate with different SNARE proteins. We also propose that coupling the guidance receptor cell machinery to proteins that critically modulate stimulus-dependent local exocytosis provides a general mechanism that links chemotropic receptor signaling to membrane turnover, thereby resulting in axonal navigation.

\section{References}

Alberts P, Rudge R, Irinopoulou T, Danglot L, Gauthier-Rouvière C, Galli T (2006) Cdc42 and actin control polarized expression of TI-VAMP vesicles to neuronal growth cones and their fusion with the plasma membrane. Mol Biol Cell 17:1194-1203.

Barallobre MJ, Del Río JA, Alcántara S, Borrell V, Aguado F, Ruiz M, Carmona MA, Martin M, Fabre M, Yuste R, Tessier-Lavigne M, Soriano E (2000) Aberrant development of hippocampal circuits and altered neural activity in netrin 1-deficient mice. Development 127:4797-4810.

Bhattacharya S, Stewart BA, Niemeyer BA, Burgess RW, McCabe BD, Lin P, Boulianne G, O'Kane CJ, Schwarz TL (2002) Members of the synaptobrevin/vesicle-associated membrane protein (VAMP) family in Drosophila are functionally interchangeable in vivo for neurotransmitter release and cell viability. Proc Natl Acad Sci U S A 99:13867-13872.

Blasi J, Chapman ER, Link E, Binz T, Yamasaki S, De Camilli P, Südhof TC, Niemann H, Jahn R (1993a) Botulinum neurotoxin A selectively cleaves the synaptic protein SNAP-25. Nature 365:160-163.

Blasi J, Chapman ER, Yamasaki S, Binz T, Niemann H, Jahn R (1993b) Botulinum neurotoxin $\mathrm{C} 1$ blocks neurotransmitter release by means of cleaving HPC-1/syntaxin. EMBO J 12:4821-4828.

Bouchard JF, Moore SW, Tritsch NX, Roux PP, Shekarabi M, Barker PA, Kennedy TE (2004) Protein kinase A activation promotes plasma membrane insertion of DCC from an intracellular pool: a novel mechanism regulating commissural axon extension. J Neurosci 24:3040-3050.

Bourikas D, Pekarik V, Baeriswyl T, Grunditz A, Sadhu R, Nardó M, Stoeckli ET (2005) Sonic hedgehog guides commissural axons along the longitudinal axis of the spinal cord. Nat Neurosci 8:297-304.

Campbell DS, Holt CE (2001) Chemotropic responses of retinal growth cones mediated by rapid local protein synthesis and degradation. Neuron 32:1013-1026.

Castillo PE, Schoch S, Schmitz F, Südhof TC, Malenka RC (2002) RIMlalpha is required for presynaptic long-term potentiation. Nature 415:327-330.

Causeret F, Hidalgo-Sanchez M, Fort P, Backer S, Popoff MR, GauthierRouvière C, Bloch-Gallego E (2004) Distinct roles of Rac1/Cdc42 and Rho/Rock for axon outgrowth and nucleokinesis of precerebellar neurons toward netrin 1. Development 131:2841-2852.

Craig AM, Wyborski RJ, Banker G (1995) Preferential addition of newly synthesized membrane protein at axonal growth cones. Nature 375: 592-594.

Danglot L, Chaineau M, Dahan M, Gendron MC, Boggetto N, Perez F, Galli T (2010) Role of TI-VAMP and CD82 in EGFR cell-surface dynamics and signaling. J Cell Sci 123:723-735. 
Deitcher D (2002) Exocytosis, endocytosis, and development. Semin Cell Dev Biol 13:71-76.

Del Río JA, González-Billault C, Ureña JM, Jiménez EM, Barallobre MJ, Pascual M, Pujadas L, Simó S, La Torre A, Wandosell F, Avila J, Soriano E (2004) MAP1B is required for Netrin 1 signaling in neuronal migration and axonal guidance. Curr Biol 14:840-850.

Dent EW, Barnes AM, Tang F, Kalil K (2004) Netrin-1 and semaphorin 3A promote or inhibit cortical axon branching, respectively, by reorganization of the cytoskeleton. J Neurosci 24:3002-3012.

de Wit H, Walter AM, Milosevic I, Gulyás-Kovács A, Riedel D, Sørensen JB, Verhage M (2009) Synaptotagmin-1 docks secretory vesicles to syntaxin-1/ SNAP-25 acceptor complexes. Cell 138:935-946.

Dulubova I, Sugita S, Hill S, Hosaka M, Fernandez I, Südhof TC, Rizo J (1999) A conformational switch in syntaxin during exocytosis: role of munc18. EMBO J 18:4372-4382.

Fazeli A, Dickinson SL, Hermiston ML, Tighe RV, Steen RG, Small CG, Stoeckli ET, Keino-Masu K, Masu M, Rayburn H, Simons J, Bronson RT, Gordon JI, Tessier-Lavigne M, Weinberg RA (1997) Phenotype of mice lacking functional Deleted in colorectal cancer (Dcc) gene. Nature 386:796-804.

Fivash M, Towler EM, Fisher RJ (1998) BIAcore for macromolecular interaction. Curr Opin Biotechnol 9:97-101.

Forcet C, Stein E, Pays L, Corset V, Llambi F, Tessier-Lavigne M, Mehlen P (2002) Netrin-1-mediated axon outgrowth requires deleted in colorectal cancer-dependent MAPK activation. Nature 417:443-447.

Fujiwara T, Mishima T, Kofuji T, Chiba T, Tanaka K, Yamamoto A, Akagawa K (2006) Analysis of knock-out mice to determine the role of HPC-1/ syntaxin 1A in expressing synaptic plasticity. J Neurosci 26:5767-5776.

Gil C, Chaib-Oukadour I, Blasi J, Aguilera J (2001) HC fragment (Cterminal portion of the heavy chain) of tetanus toxin activates protein kinase $\mathrm{C}$ isoforms and phosphoproteins involved in signal transduction. Biochem J 356:97-103.

Gitai Z, Yu TW, Lundquist EA, Tessier-Lavigne M, Bargmann CI (2003) The netrin receptor UNC-40/DCC stimulates axon attraction and outgrowth through enabled and, in parallel, Rac and UNC-115/AbLIM. Neuron 37:53-65.

Hamburger V, Hamilton HL (1992) A series of normal stages in the development of the chick embryo. 1951. Dev Dyn 195:231-272.

Hong K, Hinck L, Nishiyama M, Poo MM, Tessier-Lavigne M, Stein E (1999) A ligand-gated association between cytoplasmic domains of UNC5 and DCC family receptors converts netrin-induced growth cone attraction to repulsion. Cell 97:927-941.

Hong K, Nishiyama M, Henley J, Tessier-Lavigne M, Poo M (2000) Calcium signalling in the guidance of nerve growth by netrin-1. Nature 403:93-98.

Igarashi M, Tagaya M, Komiya Y (1997) The soluble N-ethylmaleimidesensitive factor attached protein receptor complex in growth cones: molecular aspects of the axon terminal development. J Neurosci 17:1460-1470.

Jahn R, Scheller RH (2006) SNAREs_-engines for membrane fusion. Nat Rev Mol Cell Biol 7:631-643.

Johnsson B, Löfås S, Lindquist G (1991) Immobilization of proteins to a carboxymethyldextran-modified gold surface for biospecific interaction analysis in surface plasmon resonance sensors. Anal Biochem 198:268-277.

Keino-Masu K, Masu M, Hinck L, Leonardo ED, Chan SS, Culotti JG, TessierLavigne M (1996) Deleted in Colorectal Cancer (DCC) encodes a netrin receptor. Cell 87:175-185.

Kennedy MJ, Ehlers MD (2011) Mechanisms and function of dendritic exocytosis. Neuron 69:856-875.

Kennedy TE, Serafini T, de la Torre JR, Tessier-Lavigne M (1994) Netrins are diffusible chemotropic factors for commissural axons in the embryonic spinal cord. Cell 78:425-435.

Lebrand C, Dent EW, Strasser GA, Lanier LM, Krause M, Svitkina TM, Borisy GG, Gertler FB (2004) Critical role of Ena/VASP proteins for filopodia formation in neurons and in function downstream of netrin-1. Neuron 42:37-49.

Leung KM, van Horck FP, Lin AC, Allison R, Standart N, Holt CE (2006) Asymmetrical beta-actin mRNA translation in growth cones mediates attractive turning to netrin-1. Nat Neurosci 9:1247-1256.

Li X, Saint-Cyr-Proulx E, Aktories K, Lamarche-Vane N (2002) Racl and Cdc42 but not RhoA or Rho kinase activities are required for neurite outgrowth induced by the Netrin-1 receptor DCC (deleted in colorectal cancer) in N1E-115 neuroblastoma cells. J Biol Chem 277:15207-15214.

Long H, Sabatier C, Ma L, Plump A, Yuan W, Ornitz DM, Tamada A, Murakami F, Goodman CS, Tessier-Lavigne M (2004) Conserved roles for Slit and Robo proteins in midline commissural axon guidance. Neuron 42:213-223.

Ly A, Nikolaev A, Suresh G, Zheng Y, Tessier-Lavigne M, Stein E (2008) DSCAM is a netrin receptor that collaborates with DCC in mediating turning responses to netrin-1. Cell 133:1241-1254.

Martinez-Arca S, Coco S, Mainguy G, Schenk U, Alberts P, Bouillé P, Mezzina M, Prochiantz A, Matteoli M, Louvard D, Galli T (2001) A common exocytotic mechanism mediates axonal and dendritic outgrowth. J Neurosci 21:3830-3838.

Maximov A, Tang J, Yang X, Pang ZP, Südhof TC (2009) Complexin controls the force transfer from SNARE complexes to membranes in fusion. Science 323:516-521.

Ming G, Song H, Berninger B, Inagaki N, Tessier-Lavigne M, Poo M (1999) Phospholipase C-gamma and phosphoinositide 3-kinase mediate cytoplasmic signaling in nerve growth cone guidance. Neuron 23:139-148.

Ming GL, Wong ST, Henley J, Yuan XB, Song HJ, Spitzer NC, Poo MM (2002) Adaptation in the chemotactic guidance of nerve growth cones. Nature 417:411-418.

Misura KM, Scheller RH, Weis WI (2000) Three-dimensional structure of the neuronal-Sec1-syntaxin 1a complex. Nature 404:355-362.

Molgó J, Siegel LS, Tabti N, Thesleff S (1989) A study of synchronization of quantal transmitter release from mammalian motor endings by the use of botulinal toxins type A and D. J Physiol 411:195-205.

Moore SW, Correia JP, Lai Wing Sun K, Pool M, Fournier AE, Kennedy TE (2008) Rho inhibition recruits DCC to the neuronal plasma membrane and enhances axon chemoattraction to netrin 1. Development 135:2855-2864.

Nishiyama M, Hoshino A, Tsai L, Henley JR, Goshima Y, Tessier-Lavigne M, Poo MM, Hong K (2003) Cyclic AMP/GMP-dependent modulation of $\mathrm{Ca}^{2+}$ channels sets the polarity of nerve growth-cone turning. Nature 423:990-995.

Nouvian R, Neef J, Bulankina AV, Reisinger E, Pangrsic T, Frank T, Sikorra S, Brose N, Binz T, Moser T (2011) Exocytosis at the hair cell ribbon synapse apparently operates without neuronal SNARE proteins. Nat Neurosci 14:411-413.

Osen-Sand A, Catsicas M, Staple JK, Jones KA, Ayala G, Knowles J, Grenningloh G, Catsicas S (1993) Inhibition of axonal growth by SNAP-25 antisense oligonucleotides in vitro and in vivo. Nature 364:445-448.

Pagano RE, Martin OC, Kang HC, Haugland RP (1991) A novel fluorescent ceramide analogue for studying membrane traffic in animal cells: accumulation at the Golgi apparatus results in altered spectral properties of the sphingolipid precursor. J Cell Biol 113:1267-1279.

Paglini G, Peris L, Diez-Guerra J, Quiroga S, Cáceres A (2001) The Cdk5p35 kinase associates with the Golgi apparatus and regulates membrane traffic. EMBO Rep 2:1139-1144.

Paradisi A, Mehlen P (2010) Netrin-1, a missing link between chronic inflammation and tumor progression. Cell Cycle 9:1253-1262.

Pekarik V, Bourikas D, Miglino N, Joset P, Preiswerk S, Stoeckli ET (2003) Screening for gene function in chicken embryo using RNAi and electroporation. Nat Biotechnol 21:93-96.

Perrin FE, Stoeckli ET (2000) Use of lipophilic dyes in studies of axonal pathfinding in vivo. Microsc Res Tech 48:25-31.

Pfenninger KH (2009) Plasma membrane expansion: a neuron's Herculean task. Nat Rev Neurosci 10:251-261.

Pfenninger KH, Laurino L, Peretti D, Wang X, Rosso S, Morfini G, Cáceres A, Quiroga S (2003) Regulation of membrane expansion at the nerve growth cone. J Cell Sci 116:1209-1217.

Pozas E, Pascual M, Nguyen Ba-Charvet KT, Guijarro P, Sotelo C, Chédotal A, Del Río JA, Soriano E (2001) Age-dependent effects of secreted Semaphorins $3 \mathrm{~A}, 3 \mathrm{~F}$, and $3 \mathrm{E}$ on developing hippocampal axons: in vitro effects and phenotype of Semaphorin $3 \mathrm{~A}(-/-)$ mice. Mol Cell Neurosci 18:26-43.

Ramakrishnan NA, Drescher MJ, Drescher DG (2009) Direct interaction of otoferlin with syntaxin 1A, SNAP-25, and the L-type voltage-gated calcium channel Cav1.3. J Biol Chem 284:1364-1372.

Rao SS, Stewart BA, Rivlin PK, Vilinsky I, Watson BO, Lang C, Boulianne G, Salpeter MM, Deitcher DL (2001) Two distinct effects on neurotransmission in a temperature-sensitive SNAP-25 mutant. EMBO J 20:6761-6771. 
Ren XR, Ming GL, Xie Y, Hong Y, Sun DM, Zhao ZQ, Feng Z, Wang Q, Shim S, Chen ZF, Song HJ, Mei L, Xiong WC (2004) Focal adhesion kinase in netrin-1 signaling. Nat Neurosci 7:1204-1212.

Rizo J, Südhof TC (2002) Snares and Munc18 in synaptic vesicle fusion. Nat Rev Neurosci 3:641-653.

Ruiz-Montasell B, Aguado F, Majó G, Chapman ER, Canals JM, Marsal J, Blasi J (1996) Differential distribution of syntaxin isoforms 1A and 1B in the rat central nervous system. Eur J Neurosci 8:2544-2552.

Sabo SL, McAllister AK (2003) Mobility and cycling of synaptic proteincontaining vesicles in axonal growth cone filopodia. Nat Neurosci 6:1264-1269.

Schiavo G, Matteoli M, Montecucco C (2000) Neurotoxins affecting neuroexocytosis. Physiol Rev 80:717-766.

Schulze KL, Bellen HJ (1996) Drosophila syntaxin is required for cell viability and may function in membrane formation and stabilization. Genetics 144:1713-1724.

Schulze KL, Broadie K, Perin MS, Bellen HJ (1995) Genetic and electrophysiological studies of Drosophila syntaxin-1A demonstrate its role in nonneuronal secretion and neurotransmission. Cell 80:311-320.

Serafini T, Kennedy TE, Galko MJ, Mirzayan C, Jessell TM, Tessier-Lavigne M (1994) The netrins define a family of axon outgrowth-promoting proteins homologous to C. elegans UNC-6. Cell 78:409-424.

Serafini T, Colamarino SA, Leonardo ED, Wang H, Beddington R, Skarnes WC, Tessier-Lavigne M (1996) Netrin-1 is required for commissural axon guidance in the developing vertebrate nervous system. Cell 87 : $1001-1014$

Sieber JJ, Willig KI, Kutzner C, Gerding-Reimers C, Harke B, Donnert G, Rammner B, Eggeling C, Hell SW, Grubmüller H, Lang T (2007) Anatomy and dynamics of a supramolecular membrane protein cluster. Science 317:1072-1076.

Song H, Poo M (2001) The cell biology of neuronal navigation. Nat Cell Biol 3:E81-E88.

Stoeckli ET, Landmesser LT (1995) Axonin-1, Nr-CAM, and Ng-CAM play different roles in the in vivo guidance of chick commissural neurons. Neuron 14:1165-1179.

Studier FW (2005) Protein production by auto-induction in high density shaking cultures. Protein Expr Purif 41:207-234.

Südhof TC, Rothman JE (2009) Membrane fusion: grappling with SNARE and SM proteins. Science 323:474-477.
Sutton RB, Fasshauer D, Jahn R, Brunger AT (1998) Crystal structure of a SNARE complex involved in synaptic exocytosis at $2.4 \AA$ resolution. Nature 395:347-353.

Tarrago T, Frutos S, Rodriguez-Mias RA, Giralt E (2006) Identification by 19F NMR of traditional Chinese medicinal plants possessing prolyl oligopeptidase inhibitory activity. Chembiochem 7:827-833.

Tcherkezian J, Brittis PA, Thomas F, Roux PP, Flanagan JG (2010) Transmembrane receptor DCC associates with protein synthesis machinery and regulates translation. Cell 141:632-644.

Tojima T, Akiyama H, Itofusa R, Li Y, Katayama H, Miyawaki A, Kamiguchi $\mathrm{H}$ (2007) Attractive axon guidance involves asymmetric membrane transport and exocytosis in the growth cone. Nat Neurosci 10:58-66.

Tojima T, Itofusa R, Kamiguchi H (2010) Asymmetric clathrin-mediated endocytosis drives repulsive growth cone guidance. Neuron 66:370-377.

Verhage M, Maia AS, Plomp JJ, Brussaard AB, Heeroma JH, Vermeer H, Toonen RF, Hammer RE, van den Berg TK, Missler M, Geuze HJ, Südhof TC (2000) Synaptic assembly of the brain in the absence of neurotransmitter secretion. Science 287:864-869.

Vilinsky I, Stewart BA, Drummond J, Robinson I, Deitcher DL (2002) A Drosophila SNAP-25 null mutant reveals context-dependent redundancy with SNAP-24 in neurotransmission. Genetics 162:259-271.

Wang GX, Poo MM (2005) Requirement of TRPC channels in netrin-1induced chemotropic turning of nerve growth cones. Nature 434: $898-904$.

Washbourne P, Thompson PM, Carta M, Costa ET, Mathews JR, LopezBenditó G, Molnár Z, Becher MW, Valenzuela CF, Partridge LD, Wilson MC (2002) Genetic ablation of the t-SNARE SNAP-25 distinguishes mechanisms of neuroexocytosis. Nat Neurosci 5:19-26.

Yao J, Sasaki Y, Wen Z, Bassell GJ, Zheng JQ (2006) An essential role for beta-actin mRNA localization and translation in $\mathrm{Ca}^{2+}$-dependent growth cone guidance. Nat Neurosci 9:1265-1273.

Yee KT, Simon HH, Tessier-Lavigne M, O’Leary DM (1999) Extension of long leading processes and neuronal migration in the mammalian brain directed by the chemoattractant netrin-1. Neuron 24:607-622.

Zhang HL, Eom T, Oleynikov Y, Shenoy SM, Liebelt DA, Dictenberg JB, Singer RH, Bassell GJ (2001) Neurotrophin-induced transport of a betaactin mRNP complex increases beta-actin levels and stimulates growth cone motility. Neuron 31:261-275. 